\title{
Improving the Readability of Time-Frequency and Time-Scale Representations by the Reassignment Method
}

\author{
François Auger, Member, IEEE, and Patrick Flandrin, Member, IEEE
}

\begin{abstract}
In this paper, the use of the reassignment method, first applied 15 years ago by Kodera, Gendrin, and de Villedary to the spectrogram, is generalized to any bilinear time-frequency or time-scale distribution. This method creates a modified version of a representation by moving its values away from where they are computed, so as to produce a better localization of the signal components. We first propose a new formulation of this method, followed by a thorough theoretical study of its characteristics. Its practical use for a large variety of known time-frequency and time-scale distributions is then addressed. Finally, some experimental results are reported to demonstrate the performance of this method.
\end{abstract}

\section{INTRODUCTION}

$\mathbf{T}$ IME-FREQUENCY or time-scale representations are more and more widely used for nonstationary signal analysis. They perform a mapping of a one-dimensional signal $x(t)$ into a two-dimensional function of time and frequency $\operatorname{TFR}(x ; t, \omega)$ or time and scale $\operatorname{TSR}(x ; t, a)$, in order to extract relevant informations. Among these, the spectrogram [1], [2] is probably one of the earliest, and still one of the most commonly used today. Nevertheless, the spectrogram has severe drawbacks, both theoretically, since it provides biased estimators of the signal instantaneous frequency and group delay, and practically, since the Gabor-Heisenberg inequality [1] makes a tradeoff between temporal and spectral resolutions unavoidable.

To overcome these important shortcomings, other nonstationary signal representations have been proposed among the Cohen's class [3], [4] of bilinear time-frequency energy distributions. The Wigner-Ville distribution [5], [6], the Margenau-Hill distribution [7], their smoothed versions [8]-[11], and many others with reduced cross-terms [12]-[15] are members of this class. Nearly at the same time, some authors also proposed other time-varying signal analysis tools based on a concept of scale rather than frequency, such as the scalogram [16], [17] (the squared modulus of the wavelet transform), the affine smoothed pseudo Wigner-Ville distribution [18] or the Bertrand distribution [19]. The theoretical properties and the

Manuscript received April 13, 1993; revised September 23, 1994. The associate editor coordinating the review of this paper and approving it for publication was Prof. S. D. Cabrera.

F. Auger is with the GE44-LRTI, Saint Nazaire, France.

P. Flandrin is with the Laboratoire de Physique-Recherche, Unité associée au C.N.R.S. $\mathrm{n}^{\circ} 1325$, Ecole Normale Supérieure de Lyon, 69364 Lyon cx 07 , France.

IEEE Log Number 9410284. application fields of this large variety of existing methods are now well determined, and wide-spread [8], [20]-[24].

Nevertheless, a critical point of these methods is their readability, which means both a good concentration of the signal components and no misleading interference terms. This characteristic is necessary for an easy visual interpretation of their outcomes and a good discrimination between known patterns for nonstationary signal classification tasks. For an appropriate readability, one must first choose a representation whose interference geometry matches the signal structure [25], and then correctly adjust its parameters, either empirically (with some a priori knowledge of the signal) or using automatic procedures [26]. But one may further improve the readability of a representation by means of an appropriate processing. This methodology has been addressed recently by different authors. Some of them [27]-[29] proposed to decompose the signal into elementary components, and to use the sum of the component representations as the signal representation. Others [30]-[32] perform a modification of the signal representation using image processing techniques. But this was also the purpose of the Modified Moving Window Method [33], [34] proposed by Kodera, Gendrin, and de Villedary 15 years ago. They suggested a clever modification of the spectrogram, which unfortunately remained unused because of implementation difficulties and because its efficiency was not proved theoretically.

The purpose of this paper is to show that the method they used, which will be called here the reassignment method, can be applied advantageously to all the bilinear time-frequency and time-scale representations, and can be easily computed for the most common ones. The organization of this paper is as follows: in Section II, we briefly present a new formulation of the reassignment method. Some of the properties of the resulting modified representations are also demonstrated. Section III addresses the use of this method for some well-known timefrequency representations, and Section IV for some time-scale representations. Finally, Section V provides some numerical examples demonstrating the efficiency of this method.

\section{The Reassignment Method}

\section{A. Presentation of the Reassignment Method}

Among all the bilinear time-frequency distributions, the Wigner-Ville distribution (WVD) [5], [6], defined as

$$
\mathrm{WV}(x ; t, \omega)=\int x(t+\tau / 2) \cdot x^{*}(t-\tau / 2) e^{-j \omega \tau} d \tau
$$


is one of the most interesting. In order to clarify our demonstration, we will first concentrate on this distribution. It possesses a high resolution in the time-frequency plane, and satisfies a large number of desirable theoretical properties [8], [20], [21]. Unfortunately, its use in practical applications is limited by the presence of nonnegligible cross-terms, resulting from interactions between signal components. These cross-terms may lead to an erroneous visual interpretation of the signal's time-frequency structure, and are also a hindrance to pattern recognition, since they may overlap with the searched timefrequency pattern.

Nevertheless, they can often be reduced while preserving the time and frequency shift invariance property (and possibly other interesting theoretical properties) by a two-dimensional low-pass filtering of the WVD, leading to a time-frequency representation of the Cohen's class [3], [4] which can be written as

$\operatorname{TFR}(x ; t, \omega)=\iint \phi_{\mathrm{TF}}(u, \Omega) \mathrm{WV}(x ; t-u, \omega-\Omega) d u \frac{d \Omega}{2 \pi}$.

However, this smoothing also produces a less accurate timefrequency localization of the signal components. Its shape and spread must therefore be properly determined so as to produce a suitable trade-off between good interference attenuation and good time-frequency concentration [8], [13], [20], [21]. Interesting examples of smoothings are the pseudo Wigner-Ville distribution [8], the smoothed pseudo Wigner-Ville distribution [9], and all the Reduced Interference Distributions [12]-[15].

As a complement to this smoothing, other processings can be used to improve the readability of a signal representation. Several authors [27]-[29] proposed to decompose the analyzed signal into elementary components, and to use the sum of the component representations as the signal representation. When this decomposition scheme is fitted to the analyzed signal, a relevant description including fewer cross-terms between distinct components can be obtained. Others [30]-[32], [24] tried to recognize the interference terms by their particular geometry and their oscillatory structure so as to remove them, using mainly image processing procedures. This works fairly well, except when signal components and cross-terms overlap. A third kind of signal representation processing, to which the reassignment method belongs, is to perform an increase of the signal components concentration. This method was first discovered by Kodera, Gendrin and de Villedary [33], [34], who used it only for the spectrogram. We will present below a new formulation of this method, leading to its practical use for a large family of TFR. Its use for time-scale representations will be examined later in Section IV.

The starting point of the reassignment method is (2). This expression shows that the value of a time-frequency representation at any point $(t, \omega)$ of the time-frequency plane is the sum of all the terms $\phi_{\mathrm{TF}}(u, \Omega) \mathrm{WV}(x ; t-u, \omega-\Omega)$, which can be considered as the contributions of the weighted Wigner-Ville distribution values at the neighboring points $(t-u, \omega-\Omega)$. TFR $(x ; t, \omega)$ is then the average of the signal energy located in a domain centered on $(t, \omega)$ and delimited by the essential support of $\phi_{T F}(u, \Omega)$. This averaging leads to the attenuation of the oscillating cross-terms, but also a signal components broadening. As shown in Fig. 1, the timefrequency representation can hence be nonzero on a point $(t, \omega)$ where the WVD indicates no energy, if there are some nonzero WVD values around. Therefore, one way to avoid this is to change the attribution point of this average, and to assign it to the center of gravity of these energy contributions, whose coordinates are

$$
\hat{t}(x ; t, \omega)=\text { } t-\frac{\iint u \cdot \phi_{\mathrm{TF}}(u, \Omega) \mathrm{WV}(x ; t-u, \omega-\Omega) d u \frac{d \Omega}{2 \pi}}{\iint \phi_{\mathrm{TF}}(u, \Omega) \mathrm{WV}(x ; t-u, \omega-\Omega) d u \frac{d \Omega}{2 \pi}}
$$

$$
\begin{array}{r}
\hat{\omega}(x ; t, \omega)= \\
\omega-\frac{\iint \Omega \cdot \phi_{\mathrm{TF}}(u, \Omega) \mathrm{WV}(x ; t-u, \omega-\Omega) d u \frac{d \Omega}{2 \pi}}{\iint \phi_{\mathrm{TF}}(u, \Omega) \mathrm{WV}(x ; t-u, \omega-\Omega) d u \frac{d \Omega}{2 \pi}}
\end{array}
$$

rather than to the point $(t, \omega)$ where it is computed. This reassignment leads to the construction of a modified version of this time-frequency representation, whose value at any point $\left(t^{\prime}, \omega^{\prime}\right)$ is therefore the sum of all the representation values moved to this point:

$$
\begin{aligned}
\operatorname{MTFR}\left(x ; t^{\prime}, \omega^{\prime}\right)= & \iint \operatorname{TFR}(x ; t, \omega) \delta\left(t^{\prime}-\hat{t}(x ; t, \omega)\right) \\
& \cdot \delta\left(\omega^{\prime}-\hat{\omega}(x ; t, \omega)\right) d t \frac{d \omega}{2 \pi}
\end{aligned}
$$

where $\delta(t)$ denotes the Dirac impulse. It should be noticed that the aim of the reassignment method is to improve the sharpness of the localization of the signal components by reallocating its energy distribution in the time-frequency plane. Thus, when the representation value is zero at one point, it is useless to reassign it. Expressions (3a) and (3b), which will be called the reassignment operators, have therefore neither sense nor use in this case. It should be also noticed that if the smoothing kernel $\phi_{\mathrm{TF}}(u, \Omega)$ is real-valued, the reassignment operators (3a) and (3b) are also real-valued, since the WVD is always real-valued.

\section{B. Properties of the Modified Representation}

Some basic theoretical properties of this modified representation can also be demonstrated:

1) Non Bilinearity: It should be noticed that the value at every point of the time-frequency representation is moved by means of the reassignment operators ( $3 a)$ and ( $3 b)$, which strongly depend on the signal. This causes the bilinearity to be lost. Therefore, the modified representation belongs no longer to Cohen's class of bilinear time-frequency representations.

2) Time and Frequency Shift Property: When the signal is shifted in time and/or frequency, the reassignment operators ( $3 a$ ) and (3b) are shifted alike, since they mainly depend on the ratio of particular time-frequency representations. This implies 
that the modified version preserves the time and frequency shift invariance:

$$
\begin{array}{ll}
\text { if } & y(t)=x\left(t-t_{1}\right) \cdot e^{j \omega_{1} t} \\
\text { then } & \mathrm{WV}(y ; t, \omega)=\mathrm{WV}\left(x ; t-t_{1}, \omega-\omega_{1}\right) \\
\text { and } & \hat{t}(y ; t, \omega)=\hat{t}\left(x ; t-t_{1}, \omega-\omega_{1}\right)+t_{1} \\
& \hat{\omega}(y ; t, \omega)=\hat{\omega}\left(x ; t-t_{1}, \omega-\omega_{1}\right)+\omega_{1} \\
\text { therefore } & \operatorname{MTFR}\left(y ; t^{\prime}, \omega^{\prime}\right)=\operatorname{MTFR}\left(x ; t^{\prime}-t_{1}, \omega^{\prime}-\omega_{1}\right) .
\end{array}
$$

3) Energy Conservation: It can also be shown that this energy reallocation is consistent with the energy conservation:

$$
\begin{gathered}
\iint \operatorname{MTFR}\left(x ; t^{\prime}, \omega^{\prime}\right) d t^{\prime} \frac{d \omega^{\prime}}{2 \pi}=\int|x(t)|^{2} d t \\
\text { if } \iint \phi_{\mathrm{TF}}(u, \Omega) d u \frac{d \Omega}{2 \pi}=1 .
\end{gathered}
$$

4) Perfectly Localized Chirps and Impulses: Finally, another interesting property of this method is that the reassignment of any representation performs a perfect localization for a chirp signal:

$$
\begin{array}{ll}
\text { If } & x(t)=A \cdot e^{j\left(\omega_{1} t+\alpha t^{2} / 2\right)} \\
\text { then } & \mathrm{WV}(x ; t, \omega)=2 \pi A^{2} \delta\left(\omega-\omega_{1}-\alpha t\right) \\
\text { and } & \hat{\omega}(x ; t, \omega)=\omega_{1}+\alpha \hat{t}(x ; t, \omega) \\
\text { therefore } & \operatorname{MTFR}\left(x ; t^{\prime}, \omega^{\prime}\right)=\left(\iint \operatorname{TFR}(x ; t, \omega)\right. \\
& \left.\delta\left(t^{\prime}-\hat{t}(x ; t, \omega)\right) d t \frac{d \omega}{2 \pi}\right) \delta\left(\omega^{\prime}-\omega_{1}-\alpha t^{\prime}\right)
\end{array}
$$

and for an impulse:

$$
\begin{array}{ll}
\text { If } & x(t)=A \cdot \delta\left(t-t_{1}\right) \\
\text { then } & \operatorname{WV}(x ; t, \omega)=A^{2} \delta\left(t-t_{1}\right) \\
\text { and } & \hat{t}(x ; t, \omega)=t_{1} \\
\text { therefore } & \operatorname{MTFR}\left(x ; t^{\prime}, \omega^{\prime}\right)=\left(\iint \operatorname{TFR}(x ; t, \omega)\right. \\
& \left.\delta\left(\omega^{\prime}-\hat{\omega}(x ; t, \omega)\right) d t \frac{d \omega}{2 \pi}\right) \delta\left(t^{\prime}-t_{1}\right) .
\end{array}
$$

These results prove the efficiency of this method, since however sharp or flat the representation of these signals is, its modified version given by expressions (3a), (3b), and (4) will always perfectly localize them. This is worth emphasizing, because there are very few known representations that have this property. Among the Cohen's class for instance, it can be shown [23] that only the Wigner-Ville distribution perfectly localizes a chirp signal on its instantaneous frequency law. In the next section, we will show in particular cases that other interesting properties can be transferred from a representation to its modified version.

\section{APPLICATION TO SOME}

\section{TIME-FREQUENCY REPRESENTATIONS}

In Section II, the use of the reassignment method was presented for any smoothed Wigner-Ville distribution. Various selections of smoothing kernels $\phi_{\mathrm{TF}}(u, \Omega)$ are examined in the following. In addition, it is well known [20] that several

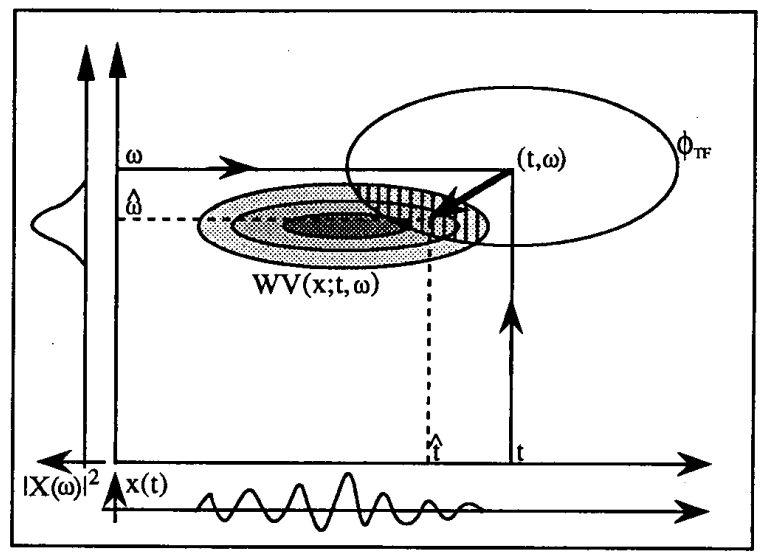

Fig. 1. Principles of the reassignment method.

Cohen's class members are not smoothed Wigner-Ville distributions. For some of them, namely the smoothed Rihaczek distributions [35], [10], [11], an appropriate implementation of the reassignment method will be also proposed.

\section{A. The Smoothed Pseudo and Pseudo Wigner-Ville Distributions}

Among all possible choices, the smoothed pseudo Wigner-Ville distribution (SPWVD) [9], [23] is one of the most versatile. Its separable kernel $\phi_{\mathrm{TF}}(u, \Omega)$ allows the time and frequency smoothings to be adjusted independently:

$$
\begin{aligned}
\phi_{\mathrm{TF}}(u, \Omega)= & g(u) H(\Omega) \\
\operatorname{SPWV}_{g, h}(x ; t, \omega)= & \iint g(u) h(\tau) x(t-u+\tau / 2) \\
& \cdot x^{*}(t-u-\tau / 2) e^{-j \omega \tau} d u d t
\end{aligned}
$$

where $g$ and $h$ are two real even windows with $h(0)=G(0)=$ 1.

To implement its modified version presents no major difficulty, since the reassignment operators (3a) and ( $3 b)$ can be computed with two additional SPWVD only:

$$
\begin{gathered}
\operatorname{MSPWV}_{g, h}\left(x ; t^{\prime}, \omega^{\prime}\right) \\
=\iint \operatorname{SPWV}_{g, h}(x ; t, \omega) \delta\left(t^{\prime}-\hat{t}(x ; t, \omega)\right) \\
\cdot \delta\left(\omega^{\prime}-\hat{\omega}(x ; t, \omega)\right) d t \frac{d \omega}{2 \pi} \\
\hat{t}(x ; t, \omega)=t-\frac{\operatorname{SPWV}_{\mathcal{T}, h}(x ; t, \omega)}{\operatorname{SPWV}_{g, h}(x ; t, \omega)} \\
\hat{\omega}(x ; t, \omega)=\omega+j \frac{\operatorname{SPWV}_{g, \mathcal{D} h}(x ; t, \omega)}{\operatorname{SPWV}_{g, h}(x ; t, \omega)} \\
\text { since } \int \Omega H(\Omega) e^{j \Omega T} \frac{d \Omega}{2 \pi}=-j \frac{d h}{d t}(t)
\end{gathered}
$$


where $\mathcal{D} h$ and $\mathcal{T} h$ are, respectively, the operators of differentiation and multiplication by the running variable:

$$
\mathcal{D} h(t)=h^{\prime}(t)=\frac{d h}{d t}(t) \text { and } \mathcal{T} h(t)=t \cdot h(t) .
$$

From a theoretical point of view, only the bilinearity of the representation is lost when the modified version is used. It is however greatly compensated by a perfect localization of chirps and impulses. Fig. 2 shows the algorithm for the computation of the MSPWVD. Compared to the SPWVD, the MSPWVD mainly requires two additional Discrete Fourier Transforms. This representation is stored in a matrix initially set to zero, where the SPWVD values are added as they are computed. At the end of the SPWVD computation, this matrix finally contains the MSPWVD.

As a particular case, the pseudo Wigner-Ville distribution (PWVD) [8] corresponds to no time smoothing

$$
\begin{aligned}
\phi_{\mathrm{TF}}(u, \Omega)= & \delta(u) H(\Omega) \\
\mathrm{PWV}_{h}(x ; t, \omega)= & \int h(\tau) x(t+\tau / 2) \cdot x^{*}(t-\tau / 2) \\
& \cdot e^{-j \omega \tau} d \tau
\end{aligned}
$$

with $h(0)=1, h^{\prime}(0)=0$. By lack of time-direction filtering, the reassignment operators are now reduced to a frequency displacement only, computed with the ratio of two particular PWVD. Therefore, the Modified PWVD values at time $t^{\prime}$ only depend on the PWVD values at the same time:

$$
\begin{aligned}
\operatorname{MPWV}_{h}\left(x ; t^{\prime}, \omega^{\prime}\right)= & \int \operatorname{PWV}_{h}\left(x ; t^{\prime}, \omega\right) \\
& \cdot \delta\left(\omega^{\prime}-\hat{\omega}\left(x ; t^{\prime}, \omega\right)\right) \frac{d \omega}{2 \pi} \\
\hat{t}(t, \omega)= & t \\
\hat{\omega}(t, \omega)= & w+j \frac{\operatorname{PWV}_{\mathcal{D} h}(x ; t, \omega)}{\operatorname{PWV}_{h}(x ; t, \omega)}
\end{aligned}
$$

From a practical point of view, this makes the computation of this modified version much faster and easier, since only one FFT is required (instead of two) and since both the PWVD and

\begin{tabular}{|c|c|}
\hline $\mathrm{X}_{\mathrm{R}}[\mathrm{k}]$ : REAL SIGNAL WITH $\mathrm{N}_{\mathbf{x}}$ POINTS. & $\mathrm{n}_{0}:$ INTTIAL TMME. \\
\hline$x[k]$ : ANALYTIC SIGNAL OF $x_{R}$. & $\Delta:$ :TIME INCREMENT \\
\hline$g[k]$ : SMOOTHING WINDOW OF 2M-1 POINTS. & \\
\hline
\end{tabular}
its modified version can be fully determined at every analysis time. This also makes the time marginal and the first order time moment of the MPWVD equal to the instantaneous power and to the instantaneous frequency:

$$
\begin{aligned}
& \text { if } x(t)=|x(t)| e^{j \varphi_{x}(t)}, \varphi_{x}^{\prime}(t)=\frac{d \varphi_{x}}{d t}(t) \\
& \text { being the signal instantaneous frequency } \\
& \int \operatorname{MPWV}_{h}\left(x ; t^{\prime}, \omega^{\prime}\right) \frac{d \omega^{\prime}}{2 \pi} \\
& =\int \operatorname{PWV}_{h}\left(x ; t^{\prime}, \omega\right) \frac{d \omega}{2 \pi}=\left|x\left(t^{\prime}\right)\right|^{2} \\
& \int \omega^{\prime} \operatorname{MPWV}_{h}\left(x ; t^{\prime}, \omega^{\prime}\right) \frac{d \omega^{\prime}}{2 \pi} \\
& =\int \hat{\omega}\left(x ; t^{\prime}, \omega\right) \operatorname{PWV}_{h}\left(x ; t^{\prime}, \omega\right) \frac{d \omega}{2 \pi} \\
& =\varphi_{x}^{\prime}\left(t^{\prime}\right)\left|x\left(t^{\prime}\right)\right|^{2} \\
& \operatorname{since}(3 . \mathrm{b}) \Rightarrow \int(\omega-\hat{\omega}(x ; t, \omega)) \mathrm{PWV}_{h}(x ; t, \omega) \frac{d \omega}{2 \pi} \\
& =\left\{\int \Omega H(\Omega) \frac{d \Omega}{2 \pi}\right\}|x(t)|^{2}=0 .
\end{aligned}
$$

D) FOR A REAL SIGNAL $X_{R}[k]$, COMPUTE FIRST ITS ANALYTIC SIGNAL $X[k]$, EITHER IN THE TTME DOMAIN, ADDING TO THE SIGNAL AN IMAGINARY PART EQUAL TO ITS HIIBERT TRANSFORM, OR IN THE FREQUENCY DOMAIN AS FOLLOWS :

- COMPUTE THE FOURIER TRANSFORM OF $X$,

- SUPPRESS THE AMPLITUDES BELONGING TO STRICTLY NEGATTVE FREQUENCIES, - DOUBLE THE AMPLTUDES OF STRICTLY POSTTIVE FREQUENCIES,

- TAKE THE INVERSE FOURIER TRANSFORM.

II) FOR A TTME-FREQUENCY REPRESENTATTON ON $\mathrm{N}_{T}$ ANALYSIS INSTANTS AND $N_{\text {TFR }}$ FREQUENCY BINS, CREATE A MATRXX MSPWV OF $\mathrm{N}_{\mathrm{T}} \times \mathrm{N}_{\text {TFR }}$ ELEMENTS, INTTALZZED TO ZERO.

II) FOR EVERY ANALYSIS TTME $n\left(n=n_{0}+i . \Delta, i=0 . . N_{T}-1\right)$ DO

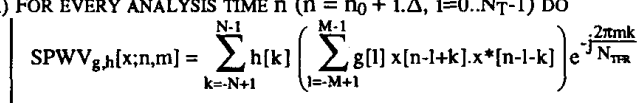

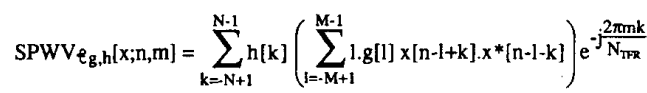

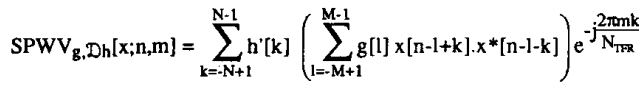

$$
\begin{aligned}
& \text { WTTH h' }[k]=T_{e} \frac{d h}{d t}\left(k T_{e}\right) \\
& \text { FOR EVERY FREQUENCY BIN } m\left(m=0 . . N_{T F R}-1\right) \text { DO } \\
& \text { IF }\left(\left|S P W V_{g, h}[x ; n, m]\right|>\varepsilon\right) \text { THEN } \\
& \hat{\mathrm{A}}[\mathrm{n}, \mathrm{m}]=\mathrm{n}-\operatorname{ROUND}\left(\frac{1}{\Delta} \frac{\mathrm{SPWV} \mathrm{e}_{\mathrm{g}}, \mathrm{h}[\mathrm{x} ; \mathrm{n}, \mathrm{m}]}{\mathrm{SPWV} \mathrm{V}_{\mathrm{g}, \mathrm{h}}[\mathrm{x} ; \mathrm{n}, \mathrm{m}]}\right) \Delta
\end{aligned}
$$

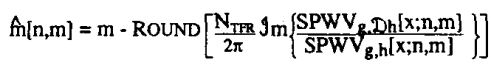

$$
\begin{aligned}
& \operatorname{MSPWV}[\hat{\mathrm{A}}, \hat{\mathrm{n}}]=\operatorname{MSPWV}[\hat{\mathrm{n}}, \hat{\mathrm{n}}]+\mathrm{SPWV}_{\mathrm{g}, \mathrm{h}}[\mathrm{x} ; \mathrm{n}, \mathrm{m}] \\
& \text { ENDIF } \\
& \text { ENDFOR } \\
& \text { ENDFOR }
\end{aligned}
$$

THE MATRIX MSPWV FNALLY CONTAINS THE MODIFED DISTRIBUTION.

Fig. 2. MSPWVD computing algorithm.

Like the MSPWVD, the MPWD retains all the properties of the PWVD except the bilinearity, which is lost for the benefit of perfectly localized chirps and impulses.

\section{B. The Reduced Interference Distributions}

The "reduced interference distributions" (RID) [12], [13], [15] are particular smoothed Wigner-Ville distributions which have recently received a considerable attention. To derive their reassignment, we will first recall that expression (2) can also be written in the following two equivalent ways:

$$
\begin{aligned}
\operatorname{TFR}(x ; t, \omega)= & \iint \phi_{\mathrm{TL}}(u, \tau) x(t-u+\tau / 2) \\
& \cdot x^{*}(t-u-\tau / 2) e^{-j \omega \tau} d u d \tau \\
= & \iint \phi_{\mathrm{DL}}(\xi, \tau) A(x ; \xi, \tau) \\
& \cdot e^{j(\xi t-\omega \tau)} \frac{d \xi}{2 \pi} d t
\end{aligned}
$$


TABLE I

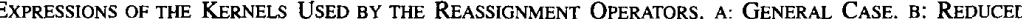
INTERFERENCE DISTRIBUTIONS, C, D: CHOI-WILUIAMS DISTRIBUTION E: F: BORN-JORDAN DISTRIBUTION

\begin{tabular}{|c|c|c|}
\hline & Ambiguity plane & \begin{tabular}{|l|} 
Time-lag plane \\
\end{tabular} \\
\hline a & $\begin{array}{l}\phi_{\mathrm{DL}}^{\hat{\mathbf{f}}}(\xi, \tau)=\mathrm{j} \frac{\partial}{\partial \xi}\left[\phi_{\mathrm{DL}}(\xi, \tau)\right] \\
\phi_{\mathrm{DL}}^{\hat{\omega}}(\xi, \tau)=-\mathrm{j} \frac{\partial}{\partial \tau}\left[\phi_{\mathrm{DL}}(\xi, \tau)\right]\end{array}$ & $\phi_{\mathrm{TL}}^{\hat{\omega}}(\mathrm{u}, \tau)=-\mathrm{j} \frac{\partial}{\partial \tau}\left[\phi_{\mathrm{TL}}(\mathrm{u}, \tau)\right]$ \\
\hline b & $\begin{array}{l}\phi_{\mathrm{DL}}^{\hat{\mathrm{f}}}(\xi, \tau)=j \tau \mathrm{F}^{\prime}(\xi \tau) \\
\phi_{\mathrm{DL}}^{\hat{\omega}}(\xi, \tau)=-j \xi F^{\prime}(\xi \tau)\end{array}$ & $\begin{array}{ll}\phi_{\mathrm{TL}}^{\hat{\mathrm{f}}}(\mathrm{u}, \tau=0)=0 & \phi_{\mathrm{TL}}^{\hat{\omega}}(\mathrm{u}, \tau=0)=0 \\
\phi_{\mathrm{TL}}^{\hat{\mathrm{f}}}(\mathrm{u}, \tau \neq 0)=\frac{\mathrm{u}}{|\tau|} \mathrm{f}(\mathrm{u} / \tau) & \phi_{\mathrm{TL}}^{\hat{\omega}}(\mathrm{u}, \tau \neq 0)=\frac{j}{|\tau|^{3}}\left(\tau . \mathrm{f}(\mathrm{u} / \tau)+\mathrm{u} \cdot \mathrm{f}^{\mathrm{f}}(\mathrm{u} / \tau)\right)\end{array}$ \\
\hline c & $\phi_{D L}(\xi, \tau)=e^{-\xi^{2} \tau^{2} / \sigma}$ & $\begin{array}{l}\phi_{\mathrm{TL}(u, \tau=0)}=\delta(u) \\
\phi_{\mathrm{TL}}(u, \tau \neq 0)=\frac{1}{\sqrt{4 \pi \tau^{2} / \sigma}} \mathrm{e}^{-\mathrm{u}^{2} /\left(4 \tau^{2} / \sigma\right)}\end{array}$ \\
\hline d & $\begin{array}{l}\phi_{D L}^{\hat{t}}(\xi, \tau)=-j \frac{2 \xi \tau^{2}}{\sigma} e^{-\xi^{2} \tau^{2} / \sigma} \\
\phi_{D L}^{\hat{\omega}}(\xi, \tau)=j \frac{2 \xi^{2} \tau}{\sigma} e^{-\xi^{2} \tau^{2} / \sigma}\end{array}$ & $\begin{array}{ll}\phi_{\mathrm{TL}}^{\hat{\mathrm{f}}(\mathrm{u}, \tau=0)=0} & \phi_{\mathrm{TL}}^{\hat{\hat{\omega}}(\mathrm{u}, \tau=0)=0} \\
\phi_{\mathrm{TL}}^{\hat{\mathrm{f}}(\mathrm{u}, \tau \neq 0)=\mathrm{u} \cdot \phi_{\mathrm{TL}}(\mathrm{u}, \tau \neq 0)} & \phi_{\mathrm{TL}}^{\hat{\hat{\omega}}}(\mathrm{u}, \tau \neq 0)=\frac{\mathrm{j} \cdot\left(2 \tau^{2}-\sigma \mathrm{u}^{2}\right)}{2 \tau \cdot|\tau|^{3} \sqrt{4 \pi / \sigma}} \mathrm{e}^{-\mathrm{u}^{2} /\left(4 \tau^{2} / \sigma\right)}\end{array}$ \\
\hline e & $\phi_{\mathrm{DL}}(\xi, \tau)=\operatorname{Sinc}(\xi \tau / 2)$ & $\begin{array}{l}\phi_{\mathrm{TL}}(\mathrm{u}, \tau=0)=\delta(\mathrm{u}) \\
\phi_{\mathrm{TL}}(\mathrm{u}, \tau \neq 0)=\frac{1}{|\tau|} \operatorname{Rect}(\mathrm{u} / \tau, 1 / 2)\end{array}$ \\
\hline f & $\begin{array}{l}\phi_{\mathrm{DL}}^{\hat{\mathrm{C}}}(\xi, \tau)=\frac{\mathrm{j} \tau}{2} \operatorname{Sinc}^{\prime}(\xi \tau / 2) \\
\phi_{\mathrm{DL}}^{\hat{\omega}}(\xi, \tau)=\frac{-\mathrm{j} \xi}{2} \operatorname{Sinc}^{\prime}(\xi \tau / 2)\end{array}$ & $\begin{array}{l}\phi_{\mathrm{TL}}^{\hat{\theta}}(\mathrm{u}, \tau=0)=0 \\
\phi_{\mathrm{TL}}^{\hat{\omega}}(\mathrm{u}, \tau \neq 0)=\frac{j}{|\tau|^{3}}[\tau \cdot \operatorname{Rect}(\mathrm{u}\{\delta(\tau, \tau / 1 / 2) \\
\end{array}$ \\
\hline & $\operatorname{Sinc}(x)=\frac{\operatorname{Sin}(x)}{x}$ & $\operatorname{Rect}(x, 1 / 2)=\left\{\begin{array}{l}1 \text { if }|x| \leq 1 / 2 \\
0 \text { if }|x|>1 / 2\end{array}\right.$ \\
\hline
\end{tabular}

where $A(x ; \xi, t)$ denotes the narrow-band ambiguity function [36] and $\phi_{\mathrm{TL}}(u, \tau), \phi_{\mathrm{DL}}(x, \tau)$ are the Fourier transforms of the smoothing kernel $\phi_{\mathrm{TF}}$ in the Doppler-lag (DL) and time-lag (TL) plane:

$$
\begin{aligned}
& A(x ; \xi, \tau)=\int x(t+\tau / 2) \cdot x^{*}(t-\tau / 2) e^{-j \xi t} d t \\
& \phi_{\mathrm{TL}}(u, \tau)=\int \phi_{\mathrm{TF}}(u, \Omega) e^{-j \Omega \tau} \frac{d \Omega}{2 \pi} \text { and } \\
& \phi_{\mathrm{DL}}(\xi, \tau)=\int \phi_{\mathrm{TL}}(u, \tau) e^{-j \xi u} d u .
\end{aligned}
$$

These expressions are often presented, because (17) clearly indicates how a time-frequency representation is computed for discrete-time signals [37], [38], and because the Doppler-lag plane is a fruitful starting point for the theoretical study of a representation [8], [20].

As shown by expressions (3a) and (3b), the reassignment operators use two particular Cohen's class members, TFR ${ }^{\hat{t}}$ and $\operatorname{TFR}^{\hat{\omega}}$, defined by expression (2) and whose smoothing kernels $\phi_{\mathrm{TF}}^{\hat{t}}(u, \Omega)$ and $\phi_{\mathrm{TF}}^{\hat{\omega}}(u, \Omega)$ are deduced from that of the reassigned representation:

$$
\hat{t}(x ; t, \omega)=t-\frac{\operatorname{TFR}^{\hat{t}}(x ; t, \omega)}{\operatorname{TFR}(x ; t, \omega)} \text { with } \phi_{\mathrm{TF}}^{\hat{t}}(u, \Omega)=u \phi_{\mathrm{TF}}(u, \Omega)
$$

$\hat{\omega}(x ; t, \omega)=\omega-\frac{\operatorname{TFR}^{\hat{\omega}}(x ; t, \omega)}{\operatorname{TFR}(x ; t, \omega)}$ with $\phi_{\mathrm{TF}}^{\hat{\omega}}(u, \Omega)=\Omega \phi_{\mathrm{TF}}(u, \Omega)$.

These two kernels can also be expressed in the Doppler-lag and time-lag planes, provided that the smoothing kernel is differentiable. These expressions are presented in Table I, line a. The "reduced interference distributions" correspond then to the particular case where $\phi_{\mathrm{DL}}(\xi, \tau)$ is a real even function of the product of the two variables:

$$
\begin{aligned}
& \phi_{\mathrm{DL}}(\xi, \tau)=F(\xi \tau) \\
& \text { with } F(0)=1 \text { and } F^{\prime}(0)=0 \\
& \left\{\begin{array}{l}
\phi_{\mathrm{TL}}(u, \tau=0)=\delta(u) \\
\phi_{\mathrm{TL}}(u, \tau \neq 0)=\frac{1}{|\tau|} f(u / \tau) .
\end{array}\right.
\end{aligned}
$$

The expressions of the corresponding $\phi_{\mathrm{TF}}^{\hat{t}}$ and $\phi_{\mathrm{TF}}^{\hat{\omega}}$ are presented in Table I, line b. Furthermore, it can be demonstrated that the structure of these kernels allows the modified version of a RID to preserve time shifts and time scalings:

if

$$
\begin{aligned}
& y(t)=\frac{1}{\sqrt{|b|}} x\left(\frac{t-t_{1}}{b}\right) \\
& \operatorname{TFR}(y ; t, \omega)=\operatorname{TFR}\left(x ; \frac{t-t_{1}}{b}, b \omega\right)
\end{aligned}
$$


and

$$
\begin{aligned}
& \hat{t}(y ; t, \omega)=b \cdot \hat{t}\left(x ; \frac{t-t_{1}}{b}, b \omega\right)+t_{1} \\
& \hat{\omega}(y ; t, \omega)=b^{-1} \cdot \hat{\omega}\left(x ; \frac{t-t_{1}}{b}, b \omega\right)
\end{aligned}
$$

therefore

$$
\operatorname{MTFR}\left(y ; t^{\prime}, \omega^{\prime}\right)=\operatorname{MTFR}\left(x ; \frac{t^{\prime}-t_{1}}{b}, b \omega^{\prime}\right) \text {. }
$$

As examples, the expressions of the two kernels $\phi_{\mathrm{TF}}^{\hat{t}}$ and $\phi_{\mathrm{TF}}^{\hat{\omega}}$ for the Choi-Williams [13] and the Born-Jordan [12] distributions (whose kernels are recalled in Table I, lines c and e) are given in Table I, lines $\mathrm{d}$ and $\mathrm{f}$, respectively.

\section{The Spectrogram}

Another classical solution is to take as a smoothing kernel the Wigner-Ville distribution of some unit energy analysis window $h(t)$. This leads to the well known spectrogram [1], [2], which is the squared modulus of the short-time Fourier transform (STFT):

$$
\begin{aligned}
\phi_{\mathrm{TF}}(u, \Omega) & =\mathrm{WV}(h ; u, \Omega) \\
S_{h}(x ; t, \omega) & =\left|\operatorname{STFT}_{h}(x ; t, \omega)\right|^{2} \quad \text { with } \operatorname{STFT}_{h}(x ; t, \omega) \\
& =\int x(u) \cdot h^{*}(t-u) e^{-j \omega u} d u .
\end{aligned}
$$

This representation is still extensively used in today's nonstationary signal analysis, although its unseparable kernel makes the spreads of the time and frequency smoothings bound, and even opposed.

The reassignment of this representation allows to run counter to its poor time-frequency concentration. In the spectrogram case, it can be shown (see the Appendix) that the reassignment operators as defined by (3a) and (3b) are also the coordinates of the center of gravity of the signal energy located in a bounded domain centered on $(t, \omega)$ and measured by the Rihaczek distribution. These coordinates are furthermore easily computed by means of short-time Fourier transforms only (see the Appendix) in the equations at the bottom of this page. Its modified version

$$
\begin{aligned}
M S_{h}\left(x ; t^{\prime}, \omega^{\prime}\right)= & \iint S_{h}(x ; t, \omega) \delta\left(t^{\prime}-\hat{t}(x ; t, \omega)\right) \\
& \cdot \delta\left(\omega^{\prime}-\hat{\omega}(x ; t, \omega)\right) d t \frac{d \omega}{2 \pi}
\end{aligned}
$$

is also nonnegative, and retains all the properties of the spectrogram except the bilinearity, still lost for the benefit of perfectly localized chirps and impulses.

Expressions (24a), (24b), (26a), (26b) are new in this paper. In the pioneering work of Kodera, Gendrin, and de Villedary [33], only expressions (25a) and (25b) were used to present the reassignment of the spectrogram. Moreover, they showed in Appendix A of [34] that this method leads to a time-frequency representation using both the squared modulus and the phase of the short-time Fourier Transform, since the reassignment operators are equal to the instantaneous frequency and group delay of the bandpass filtered signal $y(t)=\operatorname{STFT}(x ; t, \omega)$ :

$$
\begin{aligned}
\hat{t}(x ; t, \omega)= & -\frac{\partial}{\partial \omega}\left[\phi_{h}(x ; t, \omega)\right] \\
\hat{\omega}(x ; t, \omega)= & \omega+\frac{\partial}{\partial t}\left[\phi_{h}(x ; t, \omega)\right] \\
& \text { with } \operatorname{STFT}_{h}(x ; t, \omega) \\
& =M_{h}(x ; t, \omega) e^{j \phi_{h}(x ; t, \omega)} .
\end{aligned}
$$

$$
\begin{aligned}
& \hat{t}(x ; t, \omega)=t-\frac{\iint u \cdot \mathrm{WV}(h ; u, \Omega) \mathrm{WV}(x ; t-u, \omega-\Omega) d u \frac{d \Omega}{2 \pi}}{\iint \mathrm{WV}(h ; u, \Omega) \mathrm{WV}(x ; t-u, \omega-\Omega) d u \frac{d \Omega}{2 \pi}} \\
& =t-\mathcal{R}\left\{\frac{\iint u \cdot R i^{*}(h ; u, \Omega) R i(x ; t-u, \omega-\Omega) d u \frac{d \Omega}{2 \pi}}{\iint R i^{*}(h ; u, \Omega) R i(x ; t-u, \omega-\Omega) d u \frac{d \Omega}{2 \pi}}\right\} \\
& =t-\mathcal{R}\left\{\frac{\operatorname{STFT}_{\mathcal{T} h}(x ; t, \omega) \cdot \operatorname{STFT}_{h}^{*}(x ; t, \omega)}{\left|\operatorname{STFT}_{h}(x ; t, \omega)\right|^{2}}\right\} \\
& \hat{\omega}(x ; t, \omega)=\omega-\frac{\iint \Omega \cdot \mathrm{WV}(h ; u, \Omega) \mathrm{WV}(x ; t-u, \omega-\Omega) d u \frac{d \Omega}{2 \pi}}{\iint \mathrm{WV}(h ; u, \Omega) \mathrm{WV}(x ; t-u, \omega-\Omega) d u \frac{d \Omega}{2 \pi}} \\
& =\omega-\mathcal{R}\left\{\frac{\iint \Omega \cdot R i^{*}(h ; u, \Omega) R i(x ; t-u, \omega-\Omega) d u \frac{d \Omega}{2 \pi}}{\iint R i^{*}(h ; u, \Omega) R i(x ; t-u, \omega-\Omega) d u \frac{d \Omega}{2 \pi}}\right\} \\
& =\omega+\mathcal{I}_{m}\left\{\frac{\operatorname{STFT}_{\mathcal{D} h}(x ; t, \omega) \cdot \mathrm{STFT}_{h}^{*}(x ; t, \omega)}{\left|\mathrm{STFT}_{h}(x ; t, \omega)\right|^{2}}\right\} \\
& \text { with } R i(x ; t, \omega)=x(t) \cdot X^{*}(\omega) e^{-j \omega t}
\end{aligned}
$$


Generally, the STFT phase information is left unused when the spectrogram is formed, although it is known to carry a relevant description of the signal [35].

Although they are physically meaningful, expressions (28a) and $(28 \mathrm{~b})$ do not lead to an efficient implementation of the reassignment operators, since for discrete time signals the derivatives must be replaced by first order differences, and since the phase of the short-time Fourier transform must be unwrapped. This probably explains why this method remained unused. On the other hand, expressions (26a) and (26b) lead to a reliable computation of the reassignment operators.

\section{The Margenau-Hill-Spectrogram and Pseudo Margenau-Hill Distributions}

Generalizing the spectrogram, the Margenau-HillSpectrogram distribution [11], [24] is the most general expression of a Cohen's class member deduced from linear time-frequency representations. It takes the form of a product of two short-time Fourier transforms, and also amounts to a separable time and frequency filtering of the Rihaczek distribution, just like the smoothed pseudo Wigner-Ville distribution does for the Wigner-Ville distribution:

$$
\begin{aligned}
\operatorname{MHS}_{g, h}(x ; t, \omega)= & \mathcal{R}\left\{K_{g h}^{-1} \cdot \operatorname{STFT}_{g}(x ; t, \omega)\right. \\
& \left.\cdot \operatorname{STFT}_{h}^{*}(x ; t, \omega)\right\} \\
= & \mathcal{R}\left\{\iint K_{g h}^{-1} \cdot g^{*}(u) \cdot H(\Omega) \cdot e^{j \Omega u}\right. \\
& \left.\cdot \operatorname{Ri}(x ; t-u, \omega-\Omega) d u \frac{d \Omega}{2 \pi}\right\} \\
& \text { with } K_{g h}=\int h(u) \cdot g^{*}(u) d u .
\end{aligned}
$$

Nevertheless, this separable smoothing can easily achieve the nonnegativity property with two equal windows. The reassignment of this distribution will not be performed by using expressions (3a) and ( $3 b$ ), but by using the center of gravity of the neighboring Rihaczek distribution values. For two distinct windows, this point merges no longer to the one given by (3a) and (3b) as in the spectrogram case. Derivations similar to those presented in the Appendix show that it should be preferred, because it can be computed with two additional particular STFT, and because it corresponds to a separate use of the phase information of each STFT shown at the bottom of this page.

It can then be shown easily that the modified version of this distribution built with these reassignment operators

$$
\begin{aligned}
\text { MMHS }_{g, h}\left(x ; t^{\prime}, \omega^{\prime}\right) \\
=\iint \operatorname{MHS}_{g, h}(x ; t, \omega) \delta\left(t^{\prime}-\hat{t}(x ; t, \omega)\right) \\
\quad . \delta\left(\omega^{\prime}-\hat{\omega}(x ; t, \omega)\right) d t \frac{d \omega}{2 \pi}
\end{aligned}
$$

is not bilinear, satisfies the time and frequency shift invariance and the energy conservation property. It is also perfectly concentrated for impulses and sine waves (which are the only two classes of signals that the Rihaczek distribution perfectly localizes), but not for chirps.

As an interesting particular case, the pseudo Margenau-Hill distribution proposed by Hippenstiel and de Oliveira [10] corresponds to no time smoothing:

$$
\begin{aligned}
g(u)= & \delta(u) \\
\mathbf{P M H}_{h}(x ; t, \omega)= & \mathcal{R}\left\{\int H(\Omega) \cdot R i(x ; t, \omega-\Omega) \frac{d \Omega}{2 \pi}\right\} \\
= & \mathcal{R}\left\{x(t) \cdot \operatorname{STFT}_{h}^{*}(x ; t, \omega) \cdot e^{-j \omega t}\right\} \\
= & \int \frac{h(\tau)}{2}\left\{x(t) \cdot x^{*}(t-\tau)+x(t+\tau)\right. \\
& \left.\cdot x^{*}(t)\right\} e^{-j \omega \tau} d \tau
\end{aligned}
$$

with $h(u)$ real and even, with $h(0)=1$ and $h^{\prime}(0)=0$. Since there is no time direction filtering, the reassignment operators

$$
\begin{aligned}
\hat{t}(x ; t, \omega) & =t-\mathcal{R}\left\{\frac{\iint u \cdot K_{g h}^{-1} \cdot g^{*}(u) \cdot H(\Omega) \cdot e^{j \Omega u} \cdot R i(x ; t-u, \omega-\Omega) d u \frac{d \Omega}{2 \pi}}{\iint K_{g h}^{-1} \cdot g^{*}(u) \cdot H(\Omega) \cdot e^{j \Omega u} \cdot R i(x ; t-u, \omega-\Omega) d u \frac{d \Omega}{2 \pi}}\right\} \\
& =t-\mathcal{R}\left\{\frac{\mathrm{STFT}_{\mathcal{T}_{g}}(x ; t, \omega) \cdot \mathrm{STFT}_{g}^{*}(x ; t, \omega)}{\left|\mathrm{STFT}_{g}(x ; t, \omega)\right|^{2}}\right\} \\
& =-\frac{\partial}{\partial \omega}\left[\phi_{g}(x ; t, \omega)\right] \\
\hat{\omega}(x ; t, \omega) & =\omega-\mathcal{R}\left\{\frac{\iint \Omega \cdot K_{g h}^{-1} \cdot g^{*}(u) \cdot H(\Omega) \cdot e^{j \Omega u} \cdot \operatorname{Ri}(x ; t-u, \omega-\Omega) d u \frac{d \Omega}{2 \pi}}{\iint K_{g h}^{-1} \cdot g^{*}(u) \cdot H(\Omega) \cdot e^{j \Omega u} \cdot \operatorname{Ri}(x ; t-u, \omega-\Omega) d u \frac{d \Omega}{2 \pi}}\right\} \\
& =\omega+\mathcal{I m}\left\{\frac{\operatorname{STFT}{ }_{\mathcal{D} h}(x ; t, \omega) \mathrm{STFT}_{h}^{*}(x ; t, \omega)}{\left|\mathrm{STFT}_{h}(x ; t, \omega)\right|^{2}}\right\} \\
& =\omega+\frac{\partial}{\partial t}\left[\phi_{h}(x ; t, \omega)\right]
\end{aligned}
$$




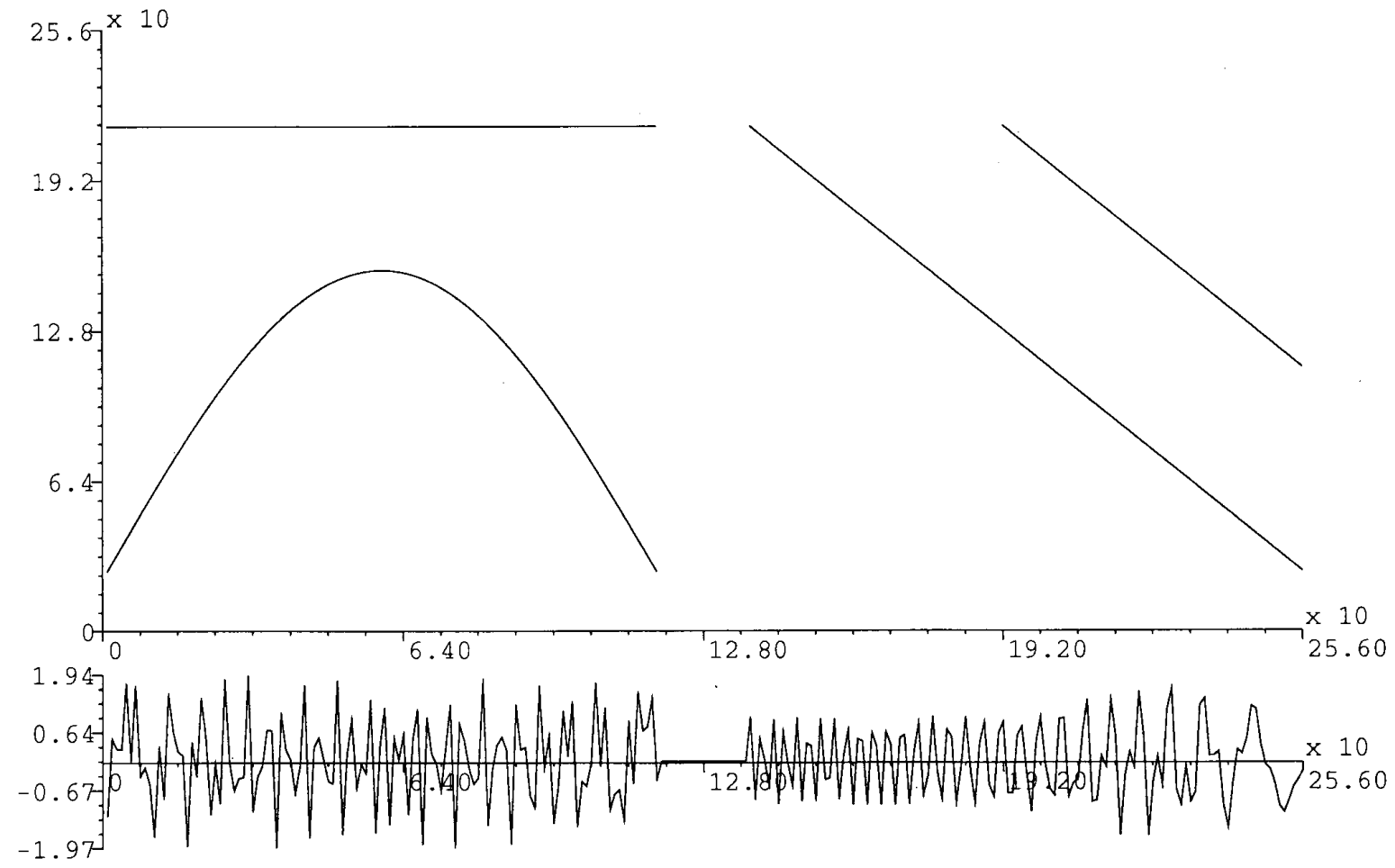

Fig. 3. Instantaneous frequency laws of the four signal components.

(31a) and (31b) yield only a frequency displacement:

$$
\begin{aligned}
\hat{t}(x ; t, \omega)= & t \\
\hat{\omega}(x ; t, \omega)= & w+\mathcal{I} m \\
& \cdot\left\{\frac{\operatorname{STFT}_{\mathcal{D} h}(x ; t, \omega) \cdot \mathbf{S T F T}_{h}^{*}(x ; t, \omega)}{\left|\operatorname{STFT}_{h}(x ; t, \omega)\right|^{2}}\right\} .
\end{aligned}
$$

This makes the implementation of the MPMHD easier than that of the MMHSD, and also leads to the preservation of both the time marginal and the first order time moment, as for the PWVD. In addition, this distribution also preserves the null values of the signal [10], [11]:

$$
\begin{aligned}
& \text { if } \exists t_{0} x\left(t_{0}\right)=0, \quad \text { then } \forall \omega^{\prime}, \\
& \operatorname{MPMH}_{h}\left(x ; t_{0}, \omega^{\prime}\right)=\mathrm{PMH}_{h}\left(x ; t_{0}, \omega^{\prime}\right)=0 .
\end{aligned}
$$

Consequently, the MPMHD retains all the properties of the PMHD except bilinearity, lost for the benefit of perfectly localized sine waves and impulses.

\section{ApPlication to Some Time-SCALE RePRESENTATIONS}

\section{A. General Case}

An interesting alternative for time-frequency analysis is provided by the recently proposed time-scale representations.
For some signal processing applications, the scale concept seems more relevant than that of frequency. One way to obtain such representations is to perform an affine smoothing of the Wigner-Ville distribution, as proposed in [18]:

$$
\begin{aligned}
\operatorname{TSR}(x ; t, a)= & \iint \phi_{\mathrm{TF}}\left(\frac{u}{a}, \Omega_{0}-a \Omega\right) \\
& \cdot \mathrm{WV}(x ; t-u, \Omega) d u \frac{d \Omega}{2 \pi}
\end{aligned}
$$

where $\Omega_{0}$ is the central frequency of the frequency-direction bandpass filtering. Since for a pure sine wave of frequency $\omega_{1}$, the time-scale distribution reaches its maximum for $a=$ $\Omega_{0} / \omega_{1}$, it is also possible to display $\operatorname{TSR}(x ; t, a)$ in a timefrequency plane by the relationship $a=\Omega_{0} / \omega$ [18].

This affine smoothing attenuates the cross-terms of the Wigner-Ville distribution and preserves now time shifts and time scalings, but of course also makes the signal components less localized. The reassignment of this representation is therefore also justified.

The starting point of its determination is expression (39), which shows that the time-scale representation value at any point $\left(t, a=\Omega_{0} / \omega\right)$ is the average of the weighted Wigner-Ville distribution values on the points $(t-v, \Omega)$ located in a domain centered on $(t, \omega)$ and bounded by the essential support of $\phi_{\mathrm{TF}}$. In order to avoid the resultant signal components broadening while preserving the crossterms attenuation, it seems once again appropriate to assign this average to the center of gravity of these energy measures, 
whose coordinates are shown in (40) at the bottom of this page, rather than to the point $\left(t, a=\Omega_{0} / \omega\right)$ where it is computed. The value of the resulting modified time-scale representation on any point $\left(t^{\prime}, a^{\prime}\right)$ is then the sum of all the representation values moved to this point:

$$
\begin{aligned}
\operatorname{MTSR}\left(x ; t^{\prime}, a^{\prime}\right)= & \iint\left(a^{\prime}\right)^{2} \operatorname{TSR}(x ; t, a) \delta\left(t^{\prime}-\hat{t}(x ; t, a)\right) \\
& \cdot \delta\left(a^{\prime}-\hat{a}(x ; t, a)\right) \frac{d t \cdot d a}{a^{2}}
\end{aligned}
$$

As was done in Section II, it can be shown easily that the modified time-scale representation is no longer bilinear, preserves time shifts and time scalings, distributes the signal energy on the whole time-scale plane, and is also perfectly localized for chirps and impulses. Three examples of particular smoothing kernels will be derived in the following.

\section{B. The Affine Smoothed Pseudo Wigner-Ville Distribution}

So as to adjust the spreads of the time and frequency smoothings independently, it is quite natural to choose a separable smoothing kernel. This leads to the affine smoothed pseudo Wigner-Ville distribution (ASPWVD) proposed in [18]:

$$
\begin{aligned}
& \phi_{\mathrm{TF}}= g(u) H(\Omega), \\
& \quad \text { with } G(0)=1 \quad \text { and } \forall \Omega \in \mathbb{R}, \\
& \int H\left(\Omega_{0}-a \Omega\right) \frac{d a}{|a|}= 1 \\
& \operatorname{ASPWV}_{g, h}(x ; t, a)= \frac{1}{|a|} \iint h(\tau / a) g(u / a) \\
& \cdot x(t-u+\tau / 2) \cdot x^{*}(t-u-\tau / 2) \\
& \cdot e^{-j \Omega_{0} \tau / a} d u d \tau .
\end{aligned}
$$

The modified version of this representation can easily be implemented, since the reassignment operators require only two additional particular ASPWVD:

$$
\begin{aligned}
\operatorname{MASPWV}_{g, h}\left(x ; t^{\prime}, a^{\prime}\right)= & \iint\left(a^{\prime}\right)^{2} \operatorname{ASPWV}_{g, h}(x ; t, a) \\
& \cdot \delta\left(t^{\prime}-\hat{t}(x ; t, a)\right) \\
& \cdot \delta\left(a^{\prime}-\hat{a}(x ; t, a)\right) \frac{d t \cdot d a}{a^{2}}
\end{aligned}
$$

$$
\begin{aligned}
\hat{t}(x ; t, a)= & t-\frac{a \cdot \operatorname{ASPWV}_{\mathcal{T}, h}(x ; t, a)}{\operatorname{ASPWV}_{g, h}(x ; t, a)} \\
\hat{\omega}(x ; t, a)= & \frac{\Omega_{0}}{\hat{a}(x ; t, a)}=\frac{\Omega_{0}}{a}+j \\
& \cdot \frac{\operatorname{ASPWV}_{g, \mathcal{D} h}(x ; t, a)}{a \cdot \operatorname{ASPWV}_{g, h}(x ; t, a)}
\end{aligned}
$$

It also retains all the properties of the ASPWVD, except bilinearity, once again lost for the benefit of perfectly localized chirps and impulses.

As a particular case, the affine pseudo Wigner-Ville distribution corresponds to no time smoothing:

$$
\begin{aligned}
\phi_{\mathrm{TF}}(u, \Omega)= & \delta(u) H(\Omega), \quad \text { satisfying } \forall \Omega \in \mathbb{R} \\
\int H\left(\Omega_{0}-a \Omega\right) \frac{d a}{|a|}= & 1 \\
\operatorname{APWV}_{h}(x ; t, a)= & \int h\left(\frac{\tau}{a}\right) x(t+\tau / 2) \cdot x^{*}(t-\tau / 2) \\
& \cdot e^{-j \Omega_{0} \tau / a} d \tau .
\end{aligned}
$$

The reassignment operators leading to its modified version yield a scale displacement only, computed with only one additional APWVD:

$$
\begin{aligned}
\operatorname{MAPWV}_{h}\left(x ; t^{\prime}, a^{\prime}\right)= & \iint\left(a^{\prime}\right)^{2} \operatorname{APWV}_{h}\left(x ; t^{\prime}, a\right) \\
& \cdot \delta\left(a^{\prime}-\hat{a}\left(x ; t^{\prime}, a\right)\right) \frac{d a}{a^{2}} \\
\hat{t}(x ; t, a)= & t \\
\hat{\omega}(x ; t, a)= & \frac{\Omega_{0}}{\hat{a}(x ; t, a)}=\frac{\Omega_{0}}{a}+j \\
& \cdot \frac{\operatorname{APWV}_{\mathcal{D} h}(x ; t, a)}{a \cdot \mathrm{APWV}_{h}(x ; t, a)}
\end{aligned}
$$

Furthermore, this representation preserves the time marginal and also leads to an estimator of the instantaneous frequency:

$$
\begin{aligned}
& \int \operatorname{MAPWV}_{h}\left(x ; t^{\prime}, a^{\prime}\right) \frac{d a^{\prime}}{a^{\prime 2}} \\
& =\int \operatorname{APWV}_{h}\left(x ; t^{\prime}, a\right) \frac{d a}{a^{2}}=\left|x\left(t^{\prime}\right)\right|^{2}
\end{aligned}
$$

$$
\begin{aligned}
& \hat{t}(x ; t, a)=t-\frac{\iint u \cdot \phi \mathrm{TF}\left(\frac{u}{a}, \Omega_{0}-a \Omega\right) \mathrm{WV}(x ; t-u, \Omega) d u \frac{d \Omega}{2 \pi}}{\iint \phi \mathrm{TF}\left(\frac{u}{a}, \Omega_{0}-a \Omega\right) \mathrm{WV}(x ; t-u, \Omega) d u \frac{d \Omega}{2 \pi}} \\
& \hat{\omega}(x ; t, a)=\frac{\Omega_{0}}{\hat{a}(x ; t, a)}=\frac{\iint \Omega \cdot \phi \mathrm{TF}\left(\frac{u}{a}, \Omega_{0}-a \Omega\right) \mathrm{WV}(x ; t-u, \Omega) d u \frac{d \Omega}{2 \pi}}{\iint \phi \operatorname{TF}\left(\frac{u}{a}, \Omega_{0}-\Omega\right) \mathrm{WV}(x ; t-u, \Omega) d u \frac{d \Omega}{2 \pi}}
\end{aligned}
$$




$$
\begin{aligned}
& \int \frac{1}{a^{\prime}} \operatorname{MAPWV}_{h}\left(x ; t^{\prime}, a^{\prime}\right) \frac{d a^{\prime}}{a^{\prime 2}} \\
& \quad=\int \frac{1}{\hat{a}(x ; t, a)} \operatorname{APWV}_{h}\left(x ; t^{\prime}, a\right) \frac{d a}{a^{2}} \\
& \quad=\frac{\varphi_{x}^{\prime}\left(t^{\prime}\right)}{\Omega_{0}}\left|x\left(t^{\prime}\right)\right|^{2} .
\end{aligned}
$$

\section{The Scalogram}

In this class of time-scale distributions, taking as smoothing kernel the WVD of some window $h(u)$ leads to the scalogram [16], [17]. This time-scale representation, which has recently become quite popular, is the squared modulus of the continuous wavelet transform:

$$
\begin{aligned}
\phi_{\mathrm{TF}}(u, \Omega)= & \mathrm{WV}(h ; u, \Omega) \\
S C_{h}(x ; t, a)= & \left|\mathrm{CWT}_{h}(x ; t, a)\right|^{2} \\
& \text { with } \mathrm{CWT}_{h}(x ; t, a)=\frac{1}{\sqrt{|a|}} \int x(u) \\
& \cdot h^{*}\left(\frac{t-u}{a}\right) e^{j \Omega_{0}(t-u) / a} d u
\end{aligned}
$$

Compared to the spectrogram, the time-frequency resolution of the scalogram depends on frequency. At high frequencies, the scalogram reaches a high time resolution but a low frequency resolution, while at low frequencies, the scalogram reaches a high frequency resolution and a low time resolution. In every case, time and frequency resolutions are still bounded by the Heisenberg-Gabor inequality, and therefore can't be both taken as small as desired.

This unsatisfactory trade-off legitimates the use of the reassignment method. As done in the Appendix for the spectrogram, it can be shown that expressions (40a) and (40b) are equivalent to the center of gravity coordinates obtained by measuring the signal energy at one point $(t, \omega)$ by means of the
Rihaczek distribution instead of the Wigner-Ville distribution. This equivalence yields an easy to compute expression of the reassignment operators, using only two particular scalograms, as shown at the bottom of this page. As for the spectrogram, the modified scalogram, defined by

$$
\begin{aligned}
\operatorname{MSC}_{h}\left(x ; t^{\prime}, a^{\prime}\right)= & \iint\left(a^{\prime}\right)^{2} S C_{h}(x ; t, a) \delta\left(t^{\prime}-\hat{t}(x ; t, a)\right) \\
& \cdot \delta\left(a^{\prime}-\hat{a}(x ; t, a)\right) \frac{d t \cdot d a}{a^{2}}
\end{aligned}
$$

is also nonnegative, and retains all the properties of the scalogram except the bilinearity, once again lost for the benefit of perfectly localized chirps and impulses.

\section{NUMERICAL EXAMPLES}

In order to evaluate the benefits of the reassignment method in practical applications, a comparison of the experimental results provided by some time-frequency representations and their modified versions is shown in this section. The analyzed signal is a 256-point computer-generated signal made up of one sine wave component, one chirp component, one chirped Gaussian packet, and one signal with constant amplitude and an instantaneous frequency describing half a sine period. Fig. 3 shows the instantaneous frequency laws of all the components, forming a time-frequency skeleton to which a time-frequency representation should be as near as possible. Fig. 4 shows the signal's WVD. The signal components are well localized, but the numerous high amplitude oscillating cross-terms make it hardly readable. Fig. 5(a) shows the PWVD (using a 79-point Gaussian window) reducing the cross-terms by a frequency direction smoothing. Its interpretation is much easier, but the signal components localization becomes coarser. Fig. 5(b) shows the modified PWVD. The improvement given by the reassignment method is obvious: all components (and also

$$
\begin{aligned}
\hat{t}(x ; t, \omega) & =t-\frac{\iint u \cdot \mathrm{WV}\left(h ; \frac{u}{a}, a \Omega\right) \cdot \mathrm{WV}(x ; t-u, \Omega) d v \frac{d \Omega}{2 \pi}}{\left|\mathrm{CWT}_{h}(x ; t, a)\right|^{2}} \\
& =t-\mathcal{R}\left\{\frac{\iint u \cdot R i^{*}\left(h ; \frac{u}{a}, a \Omega\right) \cdot R i(x ; t-u, \Omega) d u \frac{d \Omega}{2 \pi}}{\left|\mathrm{CWT}_{h}(x ; t, a)\right|^{2}}\right\} \\
\hat{\omega}(x ; t, \omega) & =\frac{\Omega_{0}}{\hat{a}(x ; t, a)}=\frac{\iint \Omega \cdot \mathrm{WV}^{2}\left(h ; \frac{u}{a}, \Omega_{0}-a \Omega\right) \cdot \mathrm{WV}(x ; t-u, \Omega) d u \frac{d \Omega}{2 \pi}}{\left|\mathrm{CWT}_{h}(x ; t, a)\right|^{2}} \\
& =t-\mathcal{R}\left\{\frac{a \cdot \mathrm{CWT}_{\mathcal{T} h}(x ; t, a) \cdot \mathrm{CWT}_{h}^{*}(x ; t, a)}{\left|\mathrm{CWT}_{h}(x ; t, a)\right|^{2}}\right\} \\
& =\mathcal{R}\left\{\frac{\iint \Omega \cdot R i^{*}\left(h ; \frac{u}{a}, \Omega_{0}-a \Omega\right) \cdot R i(x ; t-u, \Omega) d u \frac{d \Omega}{2 \pi}}{\left|\mathrm{CWT}_{h}(x ; t, a)\right|^{2}}\right\} \\
& =\frac{\Omega_{0}}{a}+\mathcal{I} m\left\{\frac{\mathrm{CWT}_{\mathcal{D} h}(x ; t, a) \cdot \mathrm{CWT}_{h}^{*}(x ; t, a)}{a\left|\mathrm{CWT}_{h}(x ; t, a)\right|^{2}}\right\}
\end{aligned}
$$




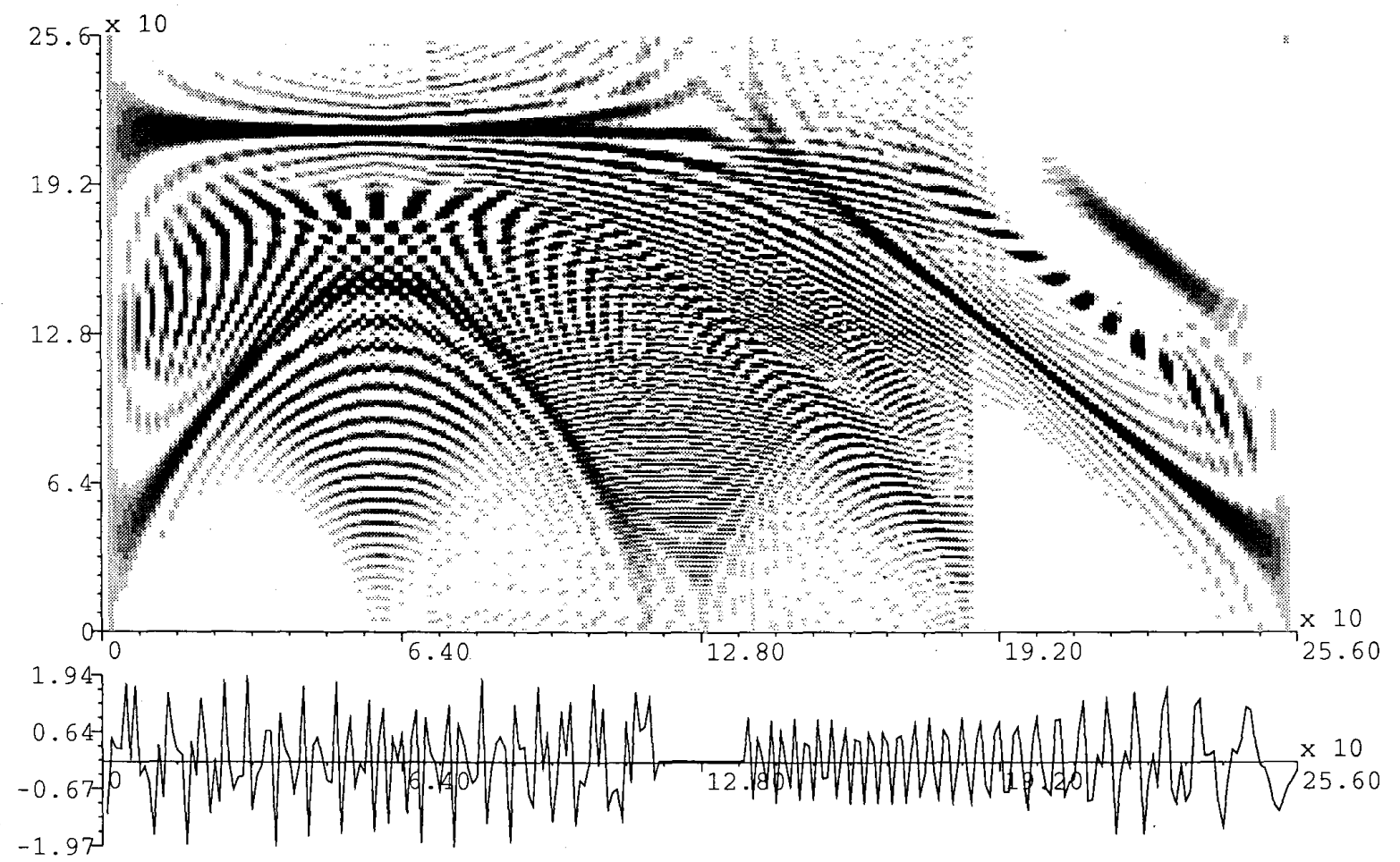

Fig. 4. Wigner-Ville distribution of the signal.

all cross-terms) are much better localized, and the sine wave and the chirp are even perfectly concentrated. Fig. 6(a) shows the SPWVD, adding a time-direction smoothing (through a 39-point Gaussian window) to the previous representation. There are very few cross-terms, but the signal components concentration is still weaker. Its modified version (shown in Fig. 6(b)) is nearly ideal: all cross-terms are removed by the smoothings, and the signal components are strongly localized by the reassignment method.

The same reasoning can also be applied to the Margenau-Hill distribution of the signal in Fig. 7. This representation is hardly readable, since its signal components concentration is worse [39] and its cross terms are twice as numerous as in the WVD. The pseudo Margenau-Hill distribution shown in Fig. 8(a) performs a frequency direction smoothing (by a 31point Gaussian window). This representation allows an easier interpretation, but still keeps some cross-terms superimposed on the signal components. Its modified version (Fig. 8(b)) gives much better localized signal components, and is even perfectly concentrated for the sine wave. The Margenau-Hill spectrogram distribution in Fig. 9(a) performs thereafter a small time direction smoothing (15-point Gaussian window) suppressing the cross-terms. A great improvement is achieved by the use of the reassignment method, leading to the modified MHSD in Fig. 9(b): all cross-terms have been removed by a 2D filtering, and the reassignment method makes the signal components strongly localized. If the time and frequency smoothing windows are equal, the representation becomes then the spectrogram (Fig. 10(a)), whose modified version
(Fig. 10(b)) perfectly localizes the chirp component. It seems more interesting for this signal to use the modified spectrogram rather than the modified MHSD, though the spectrogram yields poor results compared to the MHSD.

Finally, the next figures show time-scale representations. The affine pseudo Wigner-Ville distribution, shown on Fig. 11(a), performs a scale-invariant frequency direction smoothing of the WVD. The frequency-dependent signal concentration is clearly illustrated by the chirped component shape. Its modified version in Fig. 11(b) yields much more concentrated signal components, but still retains some crossterms. An additional scale-invariant time direction smoothing removes nearly all cross-terms, yielding an ASPWVD (Fig. 12(a)) with less concentrated signal components, and a nearly ideal MASPWVD (Fig. 12(b)). Fig. 13(a) now shows a scalogram whose window length was chosen to provide the same frequency direction smoothing, but (consequently) an approximately two times longer time direction smoothing than the previous ASPWVD (see [18, appendix D]). All the WVD cross-terms have been removed, but the time resolution is really inadequate, especially at low frequencies. Its modified version is much easier to interpret, but the localization of the component with sinusoidal frequency modulation seems weaker than on the ASPWVD.

It should also be noticed that all these figures use the same six gray-scale levels going logarithmically from the maximum value of the TFR to one hundredth of this maximum. Their readability has not been improved by a misleading display mode. 


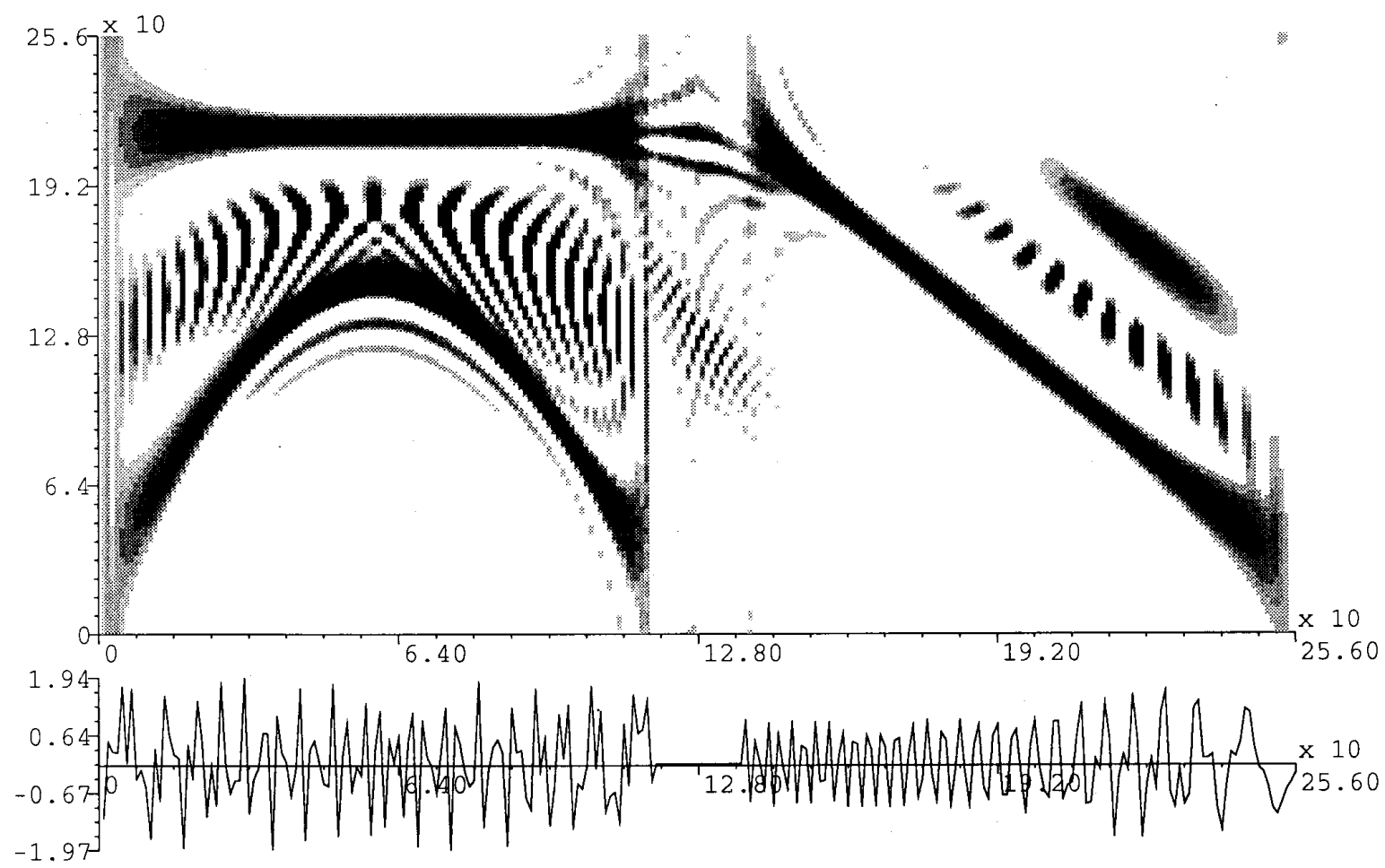

(a)

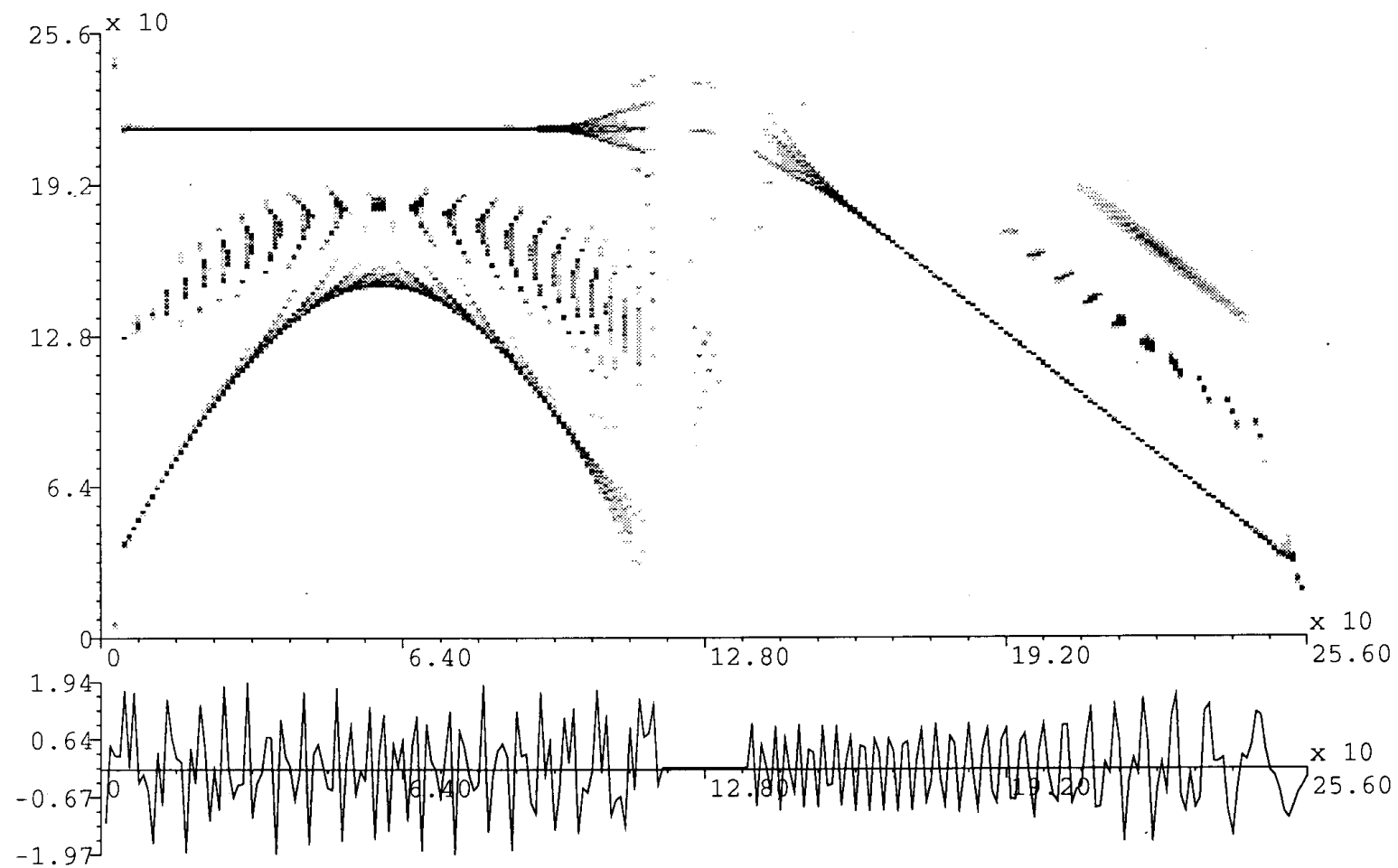

(b)

Fig. 5. (a) PWVD of the signal. $h$ : 79-point Gaussian window, (b) Modified version of the PWVD shown in (a) 


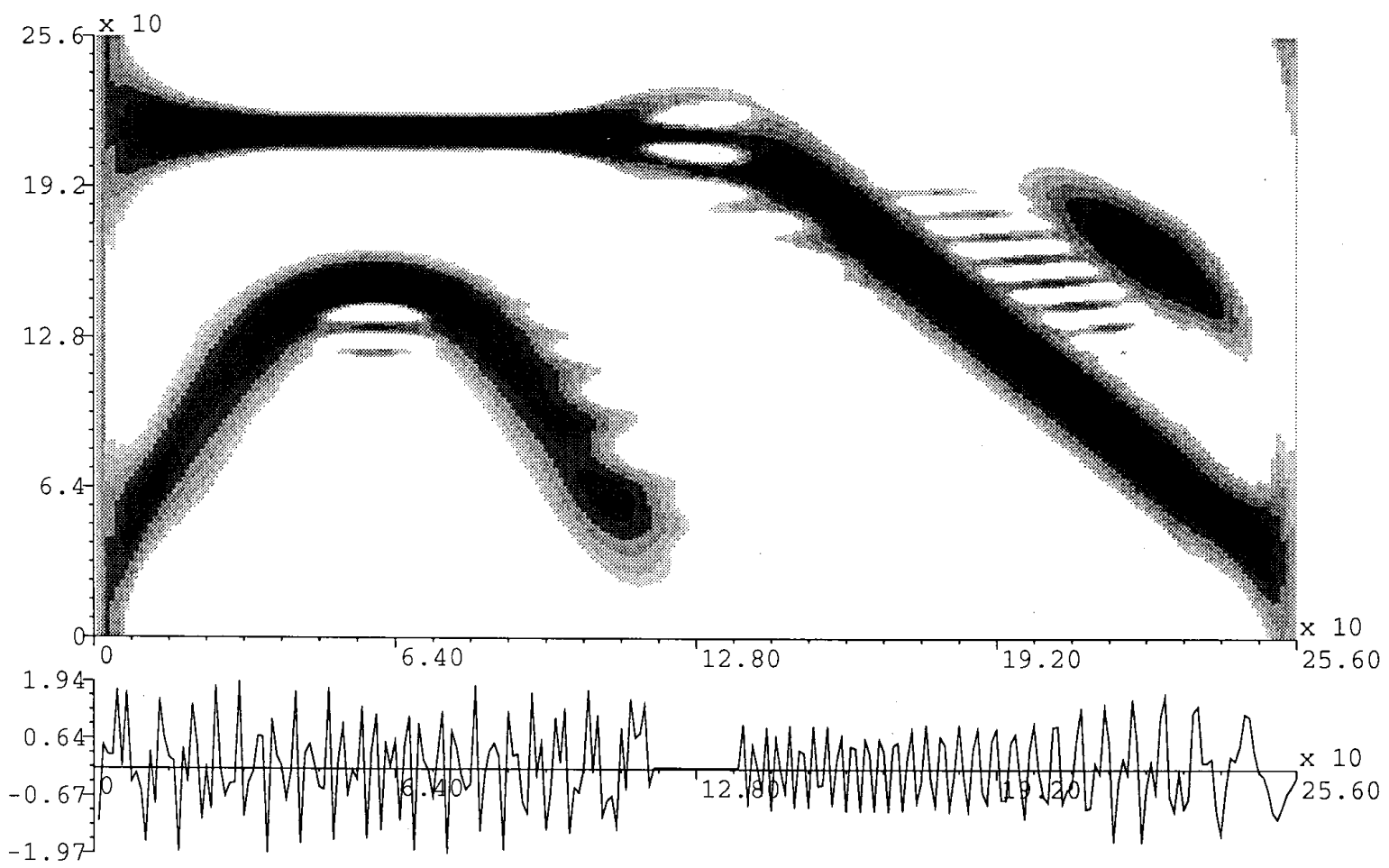

(a)

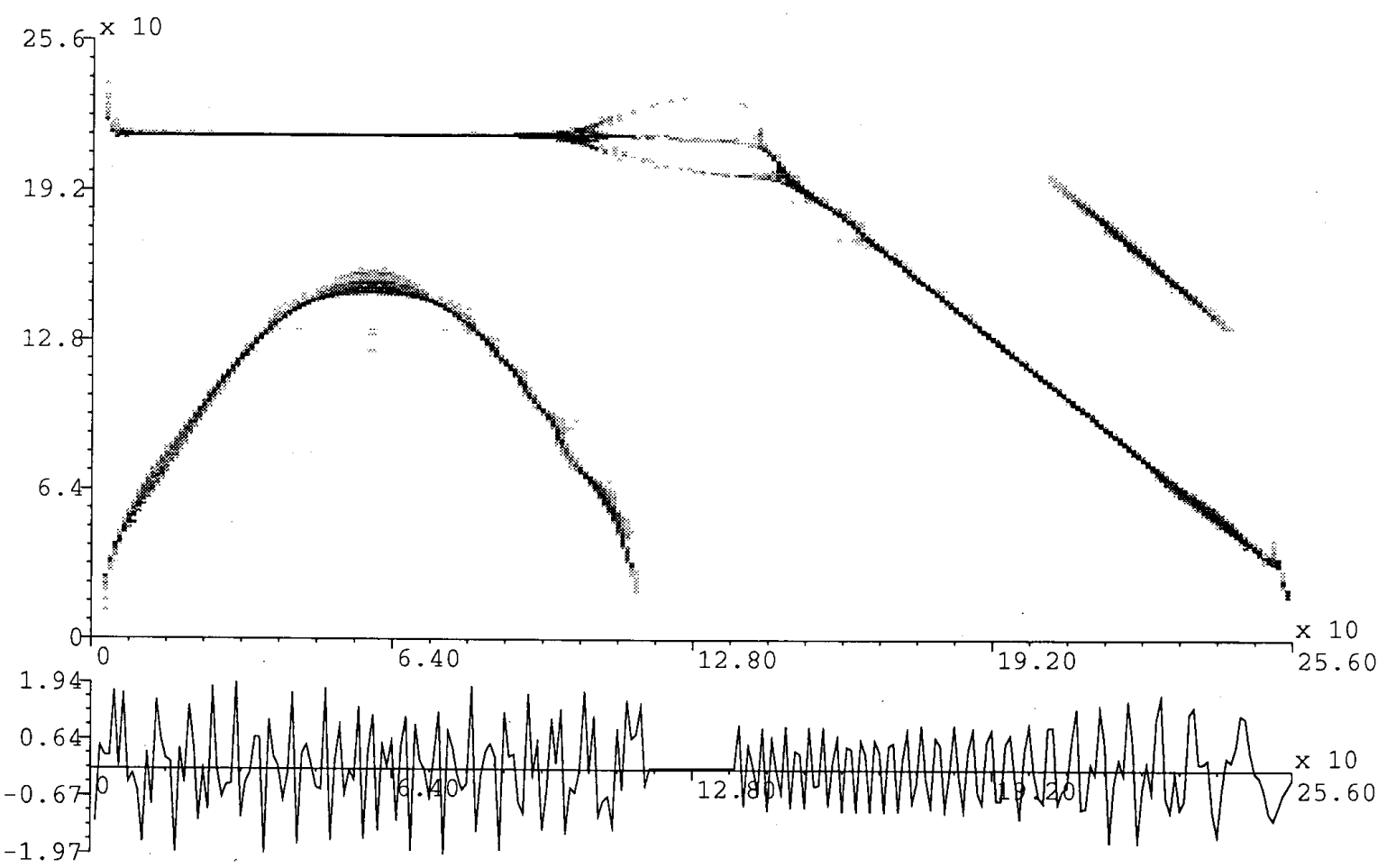

(b)

Fig. 6. (a) SPWVD of the signal. $h$ : same as Fig. 5(a); $g: 39$-point Gaussian window. (b) Modified version of the SPWVD shown in (a). 


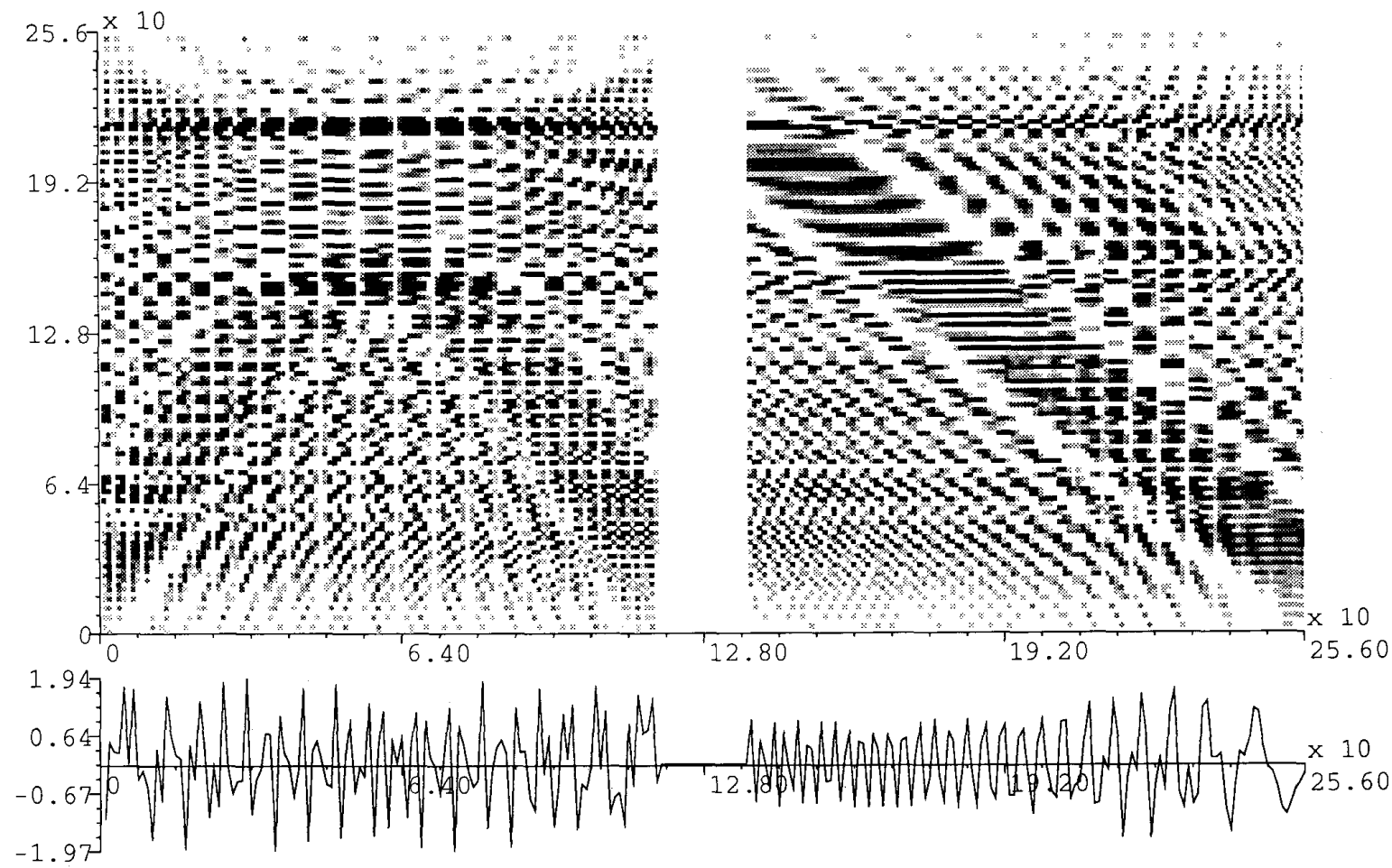

Fig. 7. Margenau-Hill distribution of the signal.

\section{CONCLUSION}

In this paper, a new method for improving the readability of time-frequency and time-scale representations has been introduced. This method creates a modified version of a representation by moving the representation values away from where they are computed. These displacements depend on the signal and on the representation, forcing the bilinearity to be lost, but they are still consistent with many of the representation properties. A comparison of the basic properties of the various representations studied here and their modified versions is presented in Table II. In the spectrogram and scalogram cases, the nonnegativity is preserved. Consequently, the reassignment method yields a whole class of time-frequency representations (the modified spectrograms) and a whole class of time-scale representations (the modified scalograms) which are nonnegative and perfectly localized for chirps and impulses. This should be emphasized, because to our knowledge there exists no other time-frequency and time-scale representations with the same properties. Another interesting point of this method is the ease of implementation. The two additional representations defined in (19a) and (19b) use the same signal values as the reassigned representation, and can therefore be computed at the same time. This requires in the worst cases two additional Fourier transforms, which is not really cumbersome.

Finally, this method should not be considered as an alternative to the representations with "signal matched" smoothing kernels [26], but as a possible (or necessary) following stage.
The experimental results reported in Section V show that the reassignment method provides a higher concentration in the time frequency plane, but of course does not remove the crossterms. Therefore, the reassignment method must be associated to a properly chosen smoothing kernel to yield simultaneously a high concentration of the signal components and a crossterms removal. The better the chosen (or designed) smoothing kernel of a representation fits the analyzed signal, the more readable its modified version.

\section{APPENDIX}

EXPRESSIONS OF THE REASSIGNMENT OPERATORS FOR THE SPECTROGRAM

From the equality:

$$
\begin{aligned}
\operatorname{STFT}_{g} & (x ; t, \omega) \cdot \operatorname{STFT}_{h}^{*}(x ; t, \omega) \\
= & \iint \mathrm{WV}(h \cdot g ; u, \Omega) \\
& \cdot \mathrm{WV}(x ; t-u, \omega-\Omega) d u \frac{d \Omega}{2 \pi}
\end{aligned}
$$

one can deduce that:

$$
\begin{aligned}
\mathcal{R}\left\{\iint u \cdot R i^{*}(h ; u, \Omega) \cdot R i(x ; t-u, \omega-\Omega) d u \frac{d \Omega}{2 \pi}\right\} \\
=\mathcal{R}\left\{\left(\int u h^{*}(u) \cdot x(t-u) \cdot e^{-j \omega(t-u)} d u\right)\right. \\
\left.\cdot\left(\int H(\Omega) \cdot X^{*}(\omega-\Omega) e^{j \Omega t} \frac{d \Omega}{2 \pi}\right)\right\}
\end{aligned}
$$




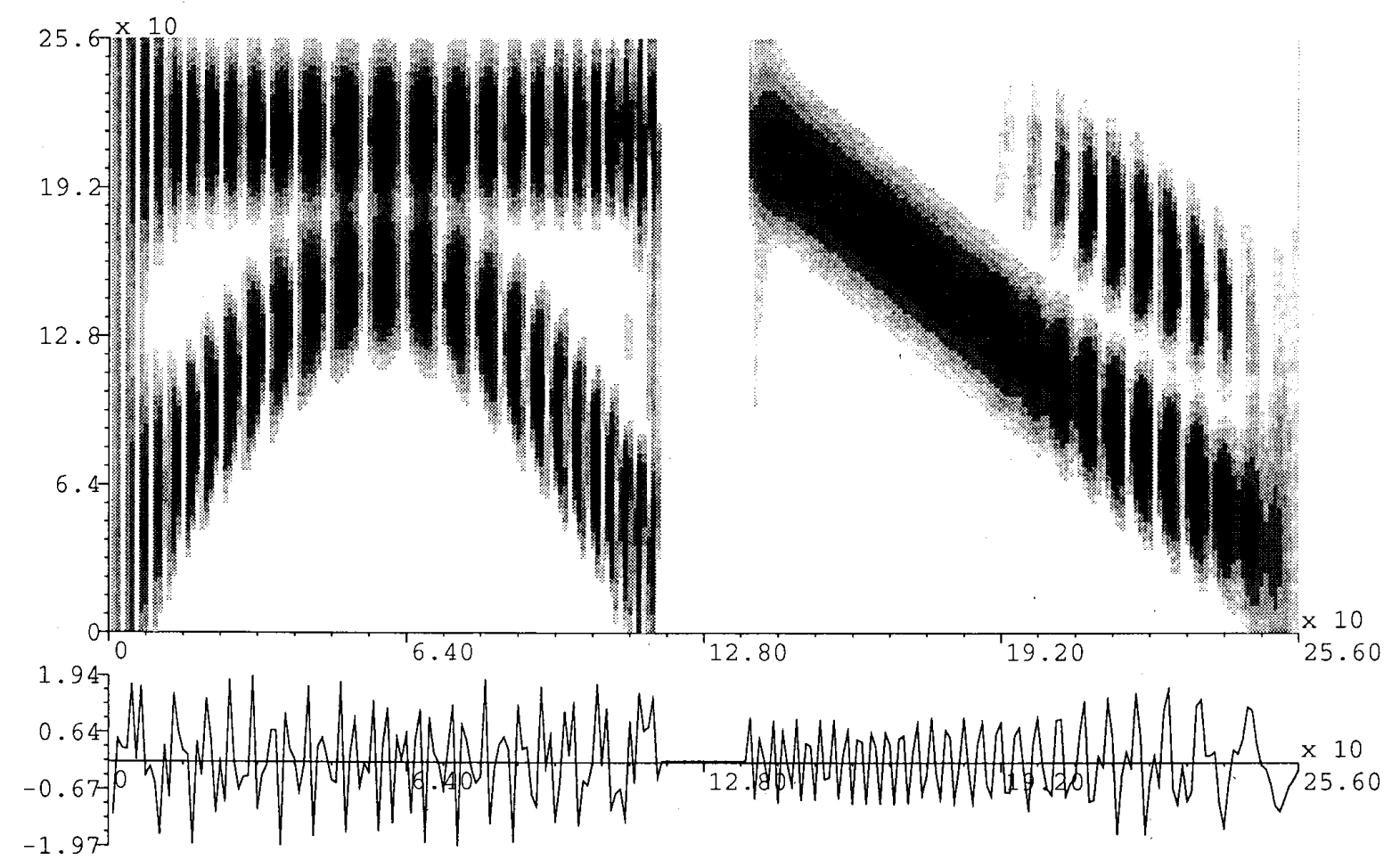

(a)

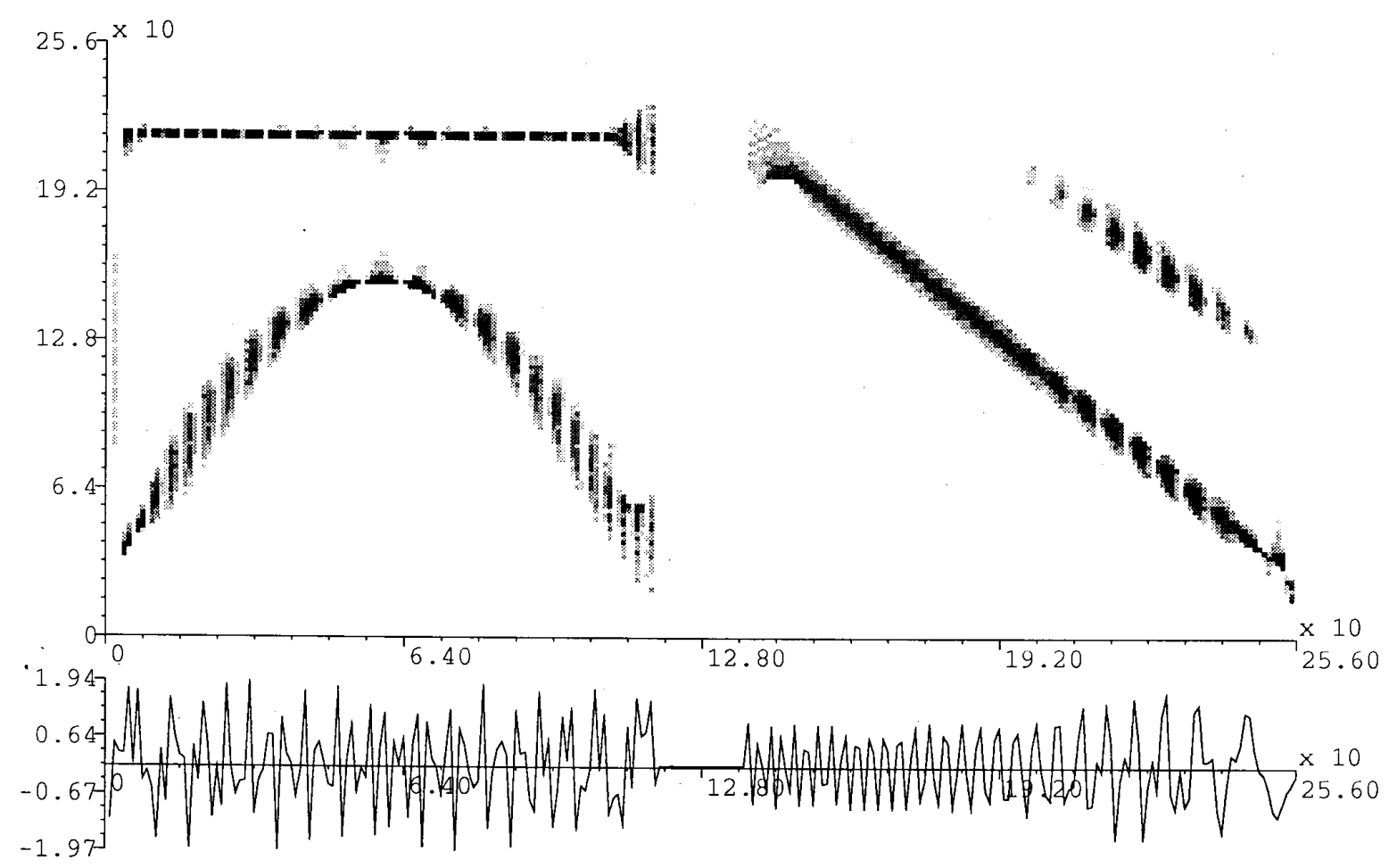

(b)

Fig. 8. (a) PMHD of the signal, $h$ : 31-point Gaussian window. (b) Modified version of the PMHD shown in (a). 

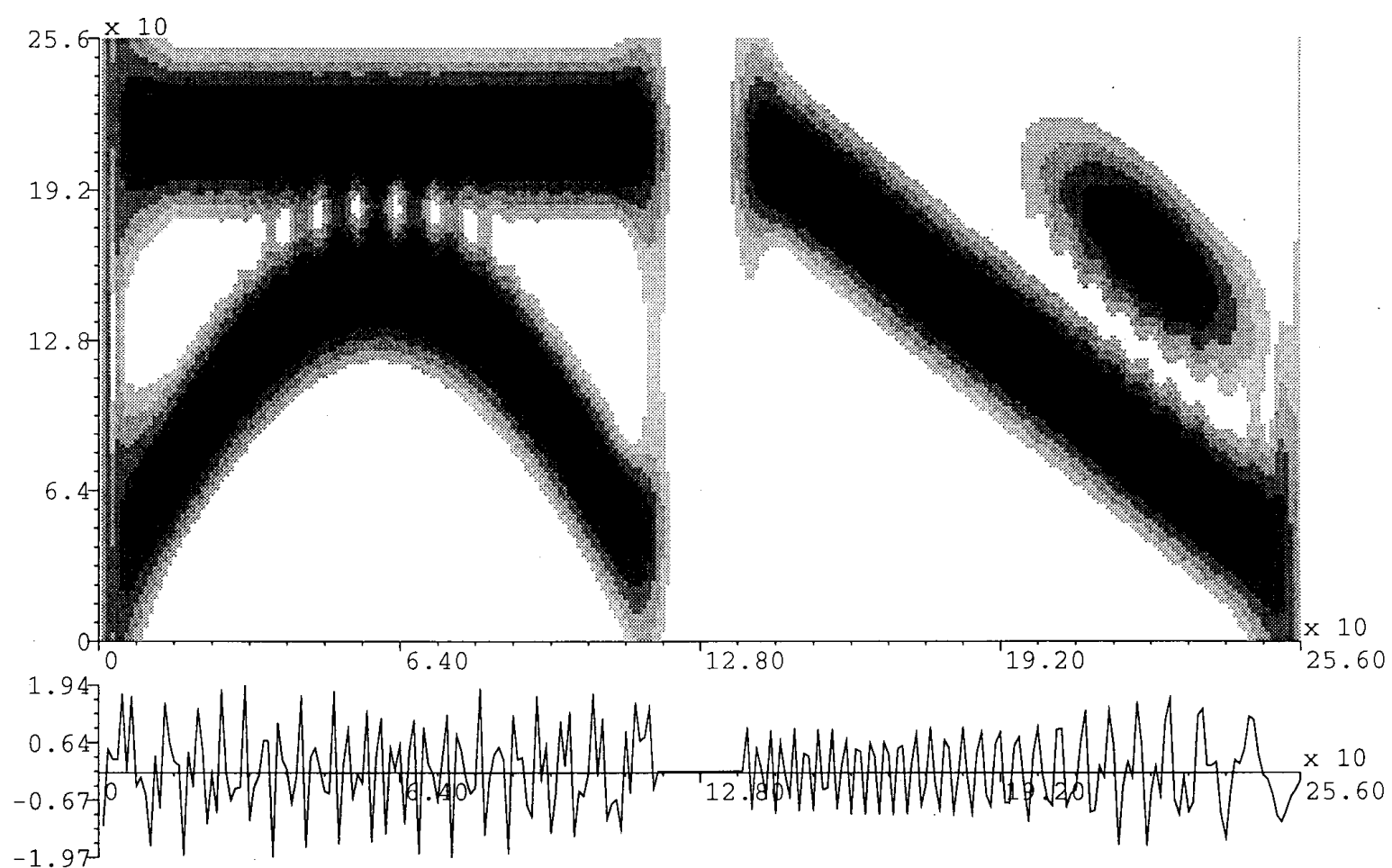
$-1.97$

(a)

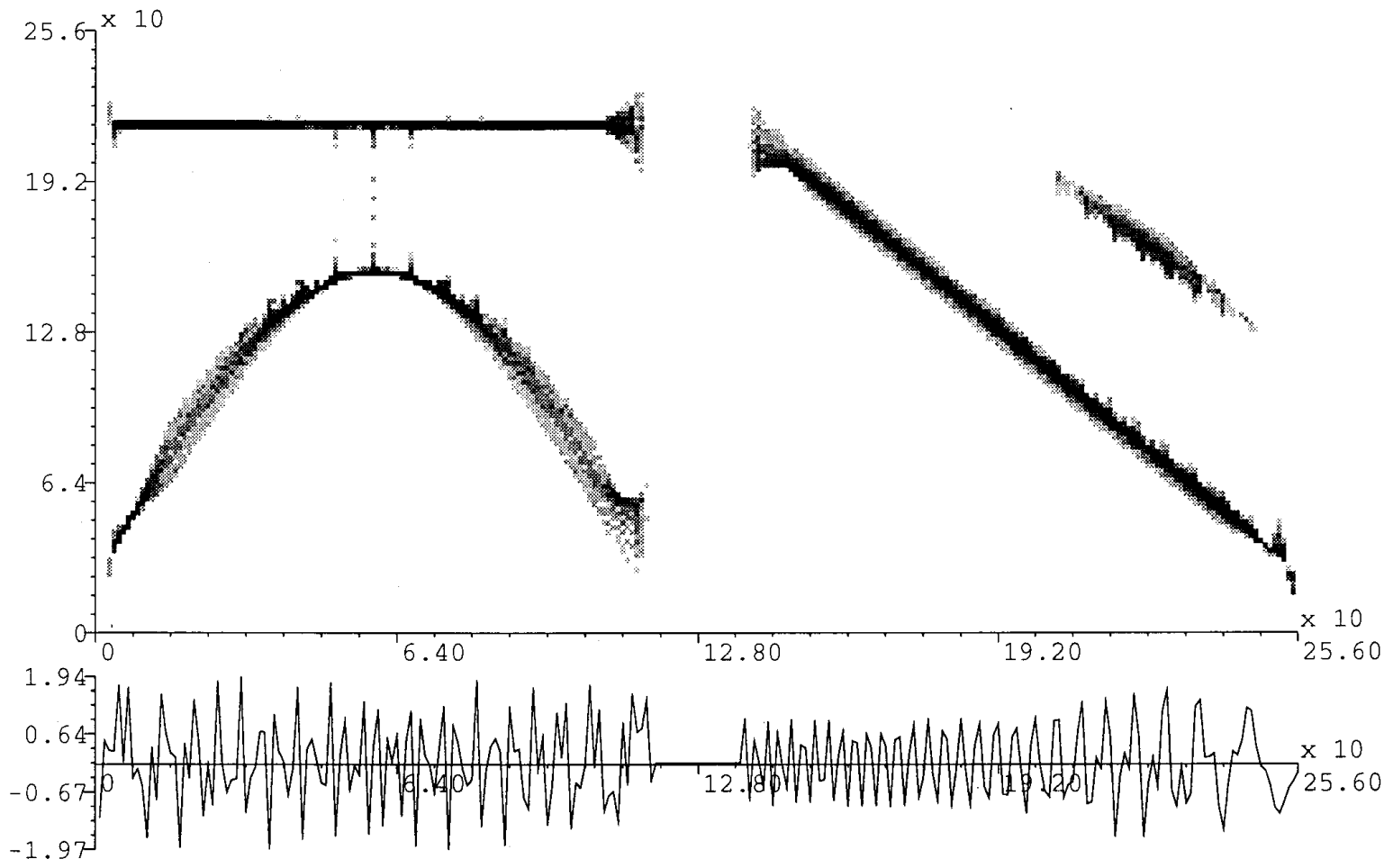

(b)

Fig. 9. (a) MHSD of the signal. $h$ : same as Fig. 8; $g: 15$-point Gaussian window. (b) Modified version of the MHSD in (a). 

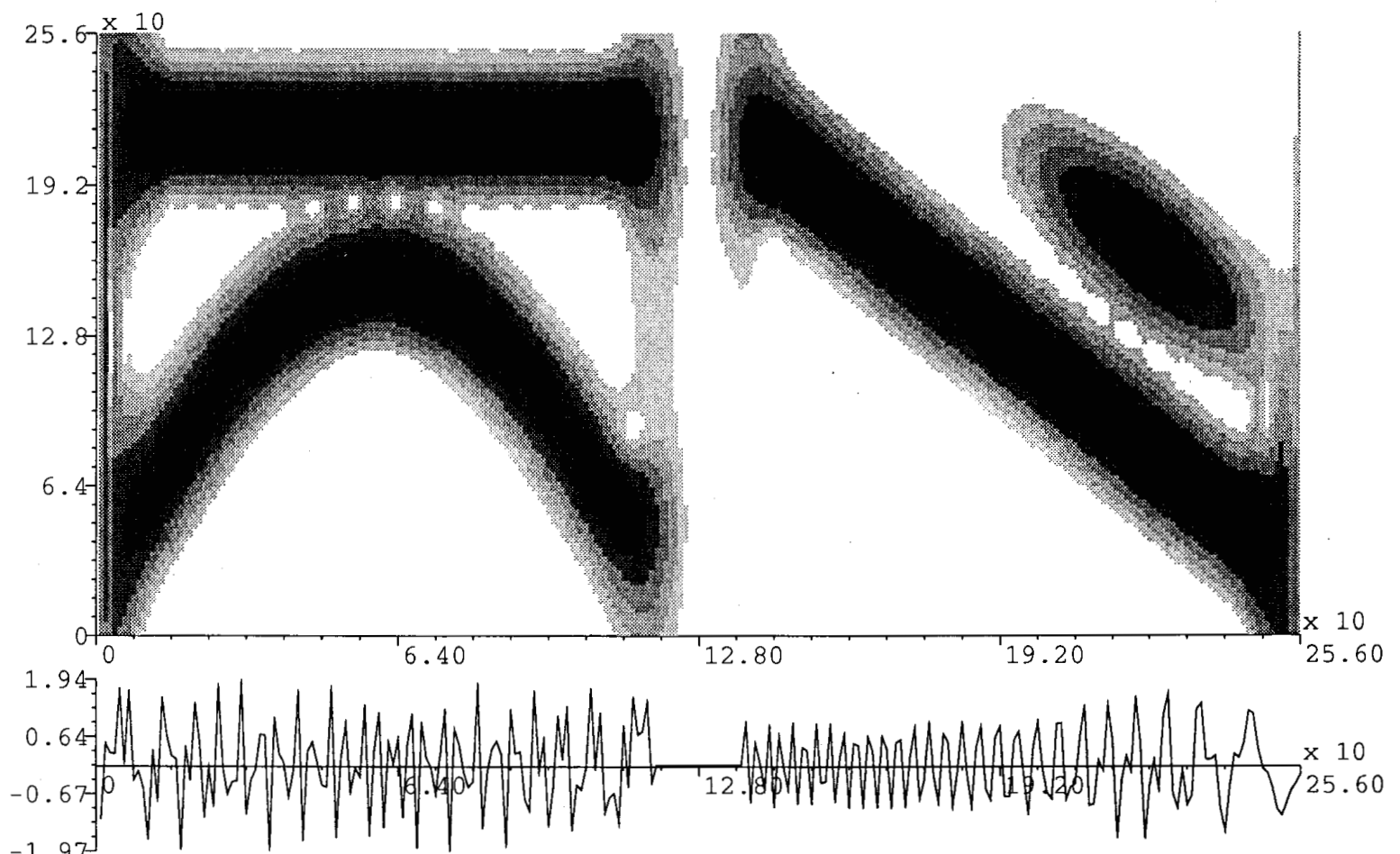
$-1.97$

(a)
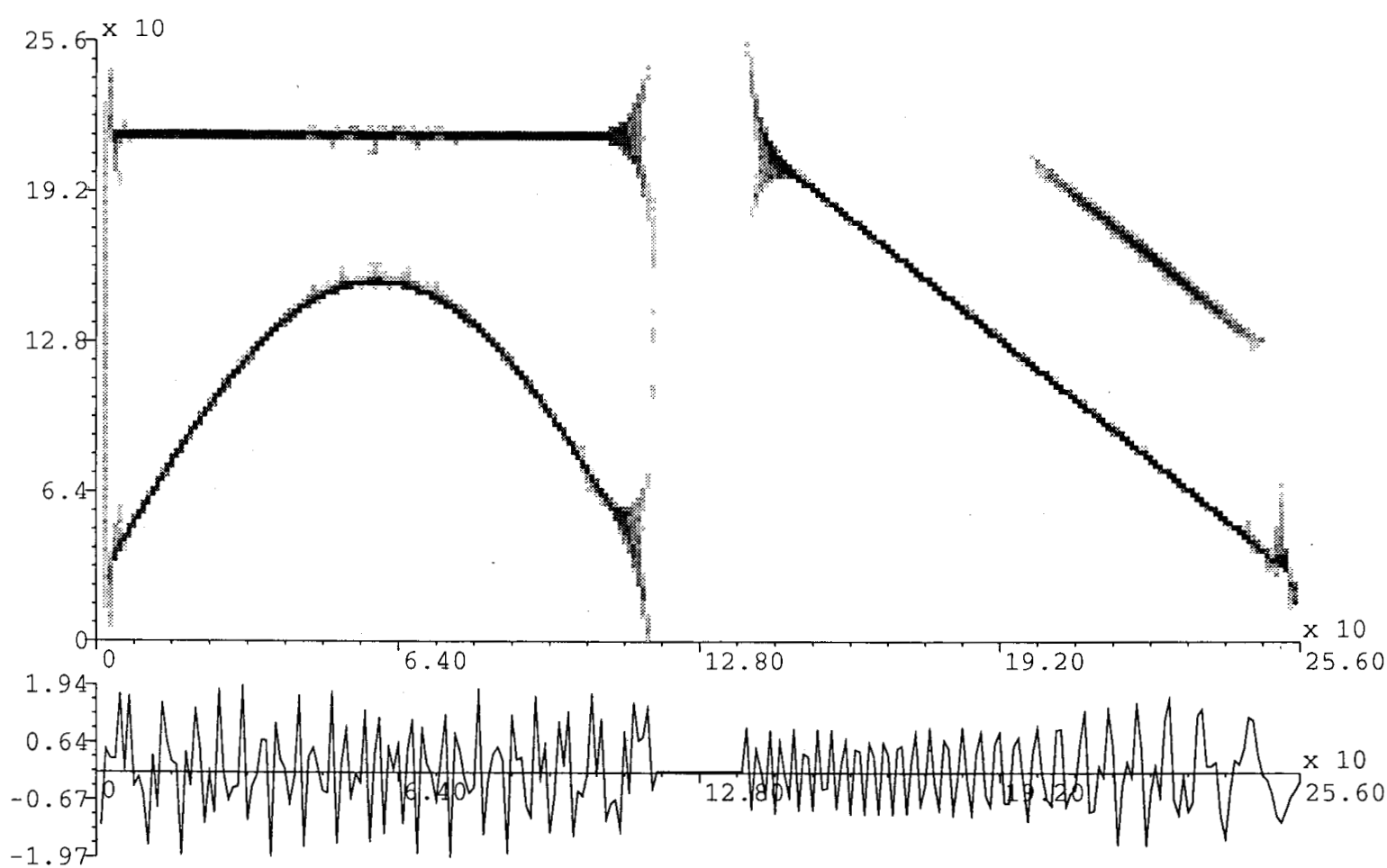

(b)

Fig. 10. (a) Spectrogram of the signal. $h: 31$-point Gaussian window. (b) Modified version of the spectrogram shown in (a). 


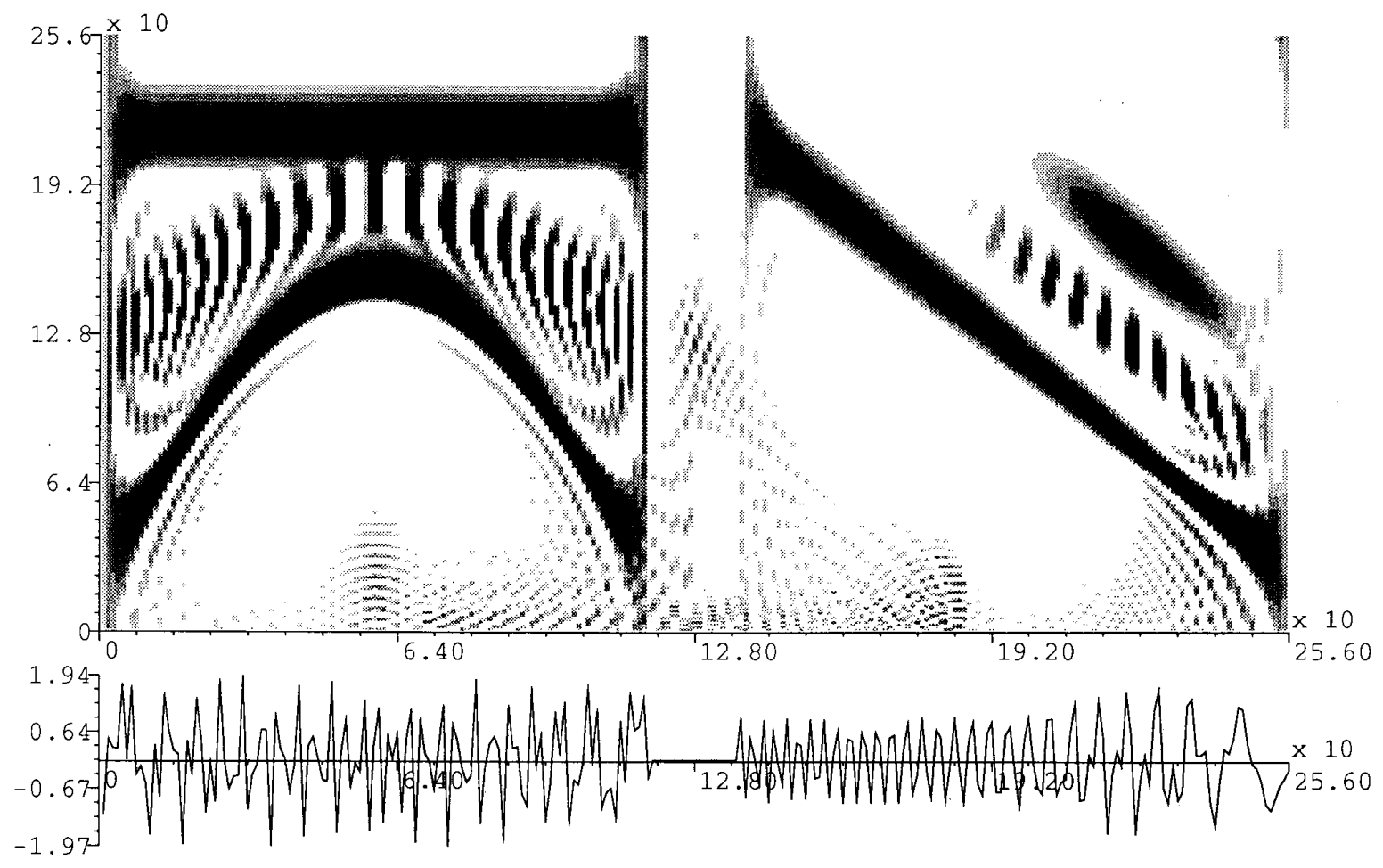

(a)

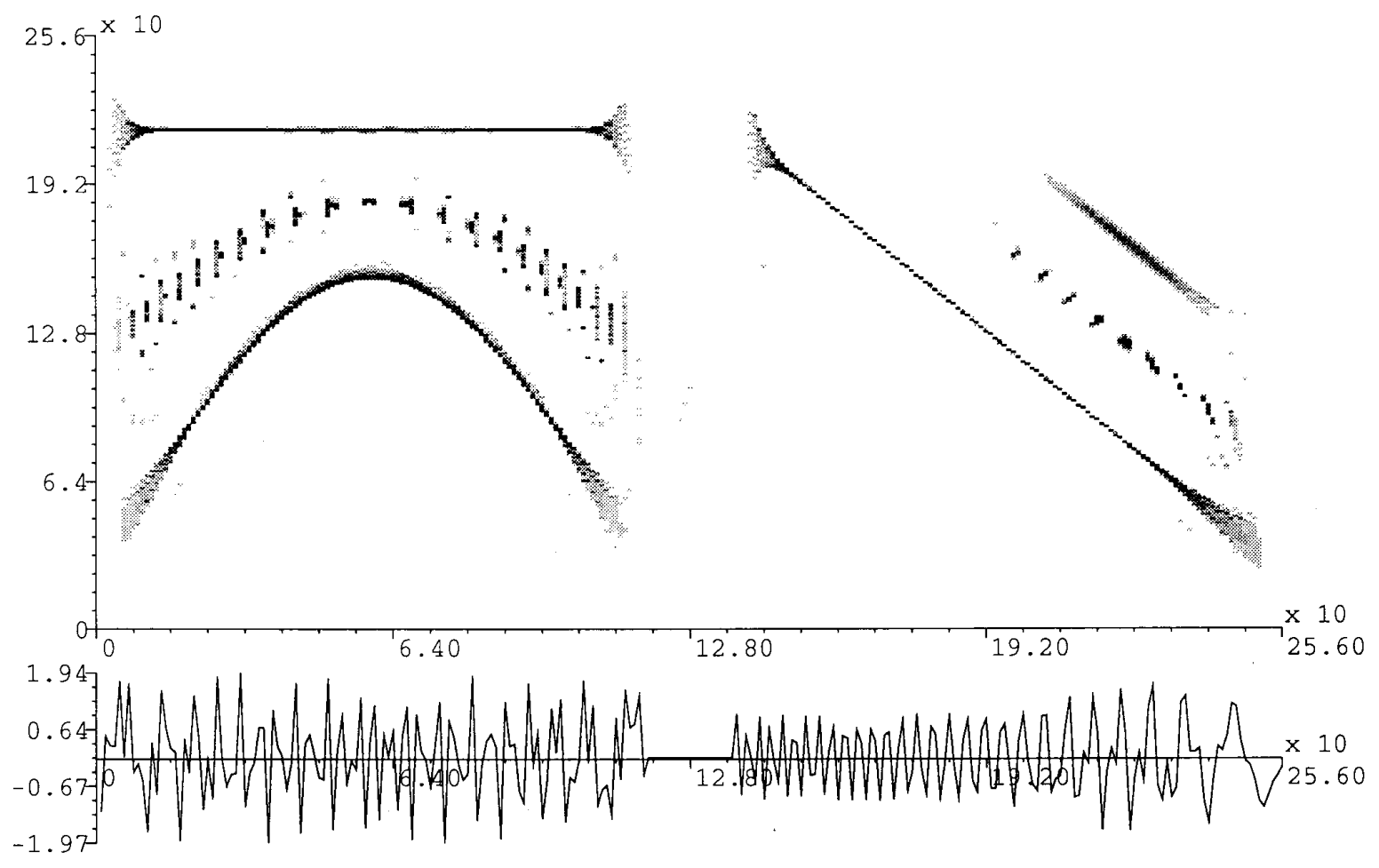

(b)

Fig. 11. (a) APWVD of the signal. $h$ : Gaussian window with $F_{0} \cdot T h=4.24$. (b) Modified version of the APWVD shown in (a). 


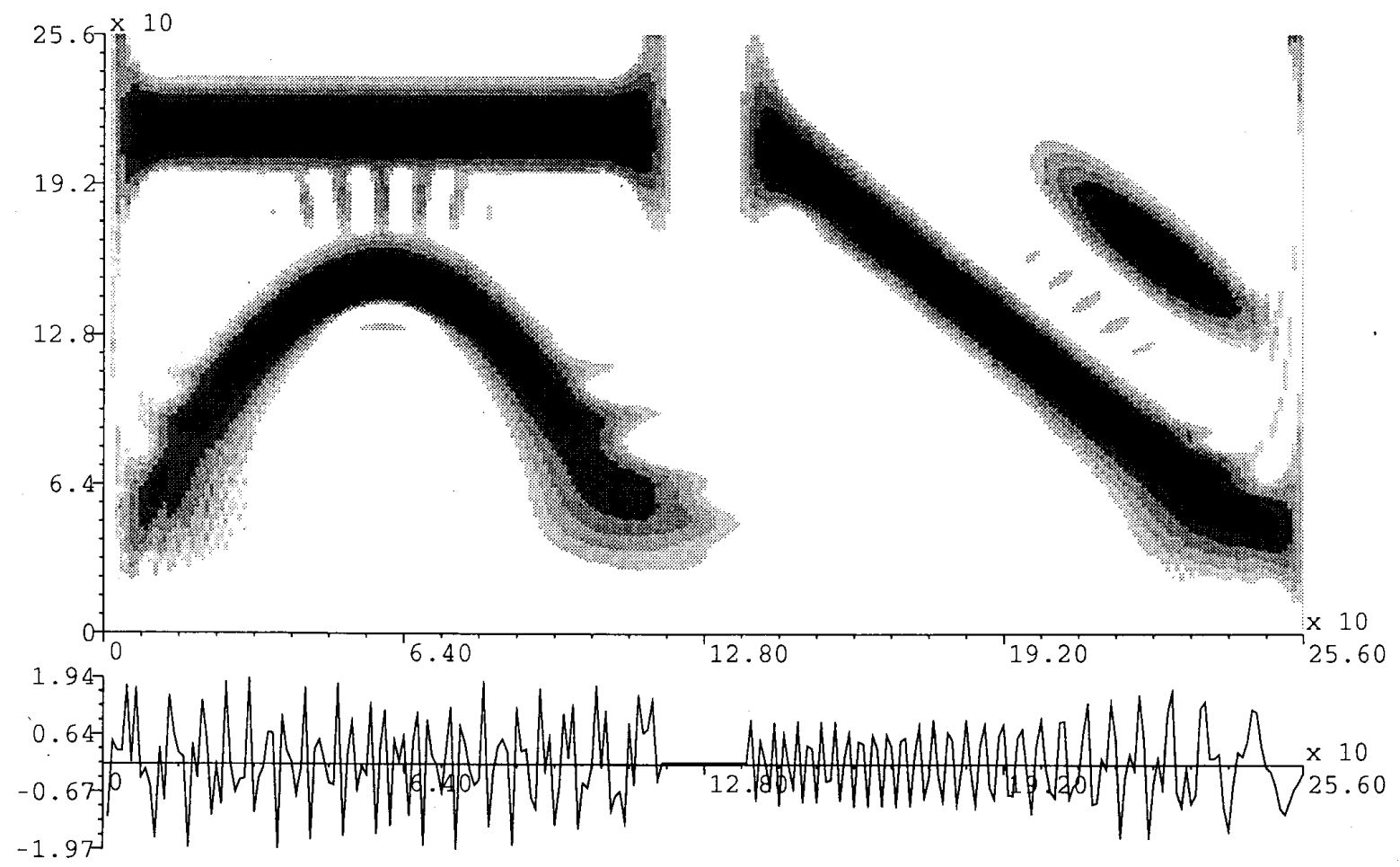

(a)

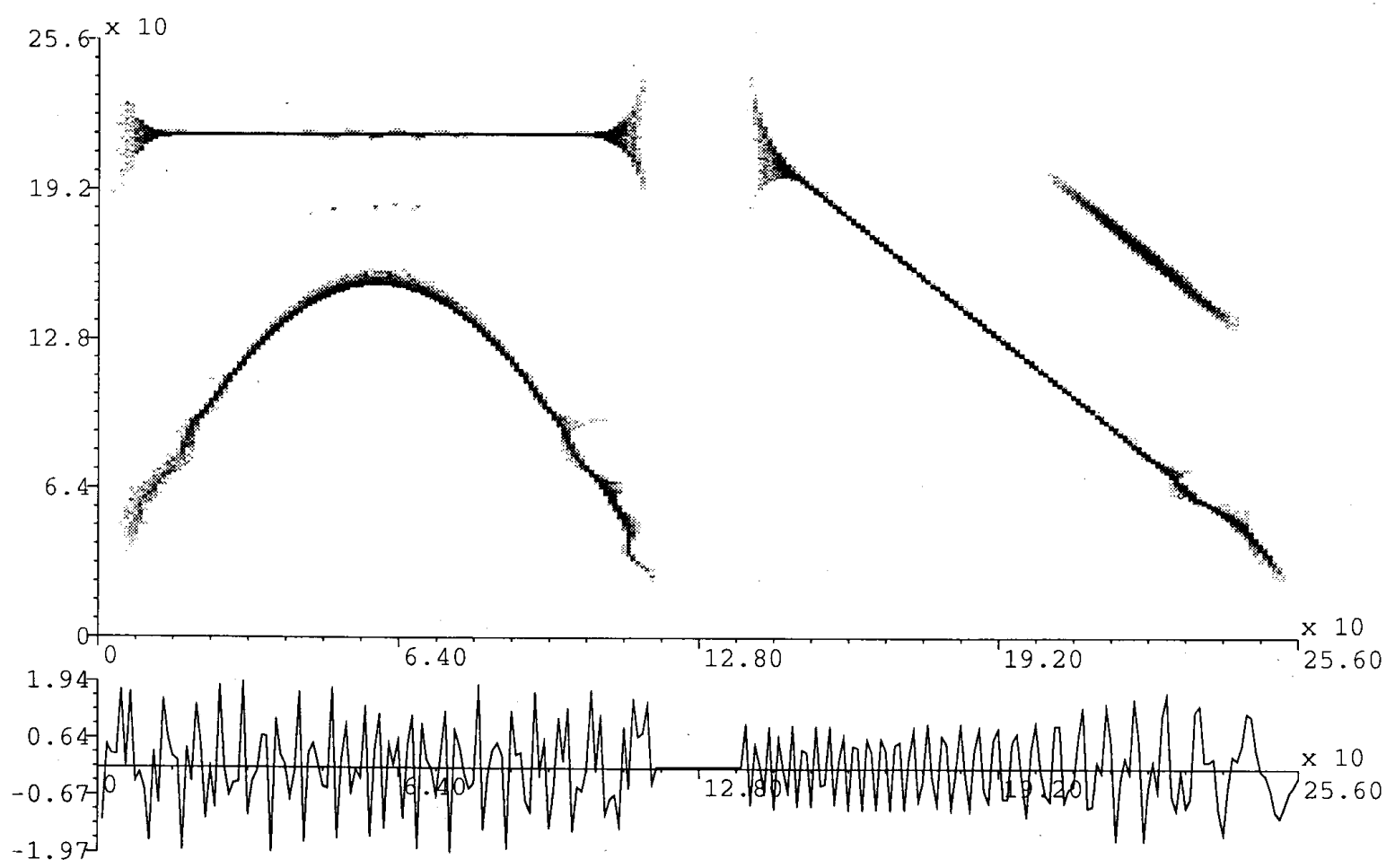

(b)

Fig. 12. (a) ASPWVD of the signal. $h$ : same as Fig. $11 ; g$ : Gaussian window with $F_{0} \cdot T g=1.0$. (b) Modified version of the ASPWVD shown in (a) 


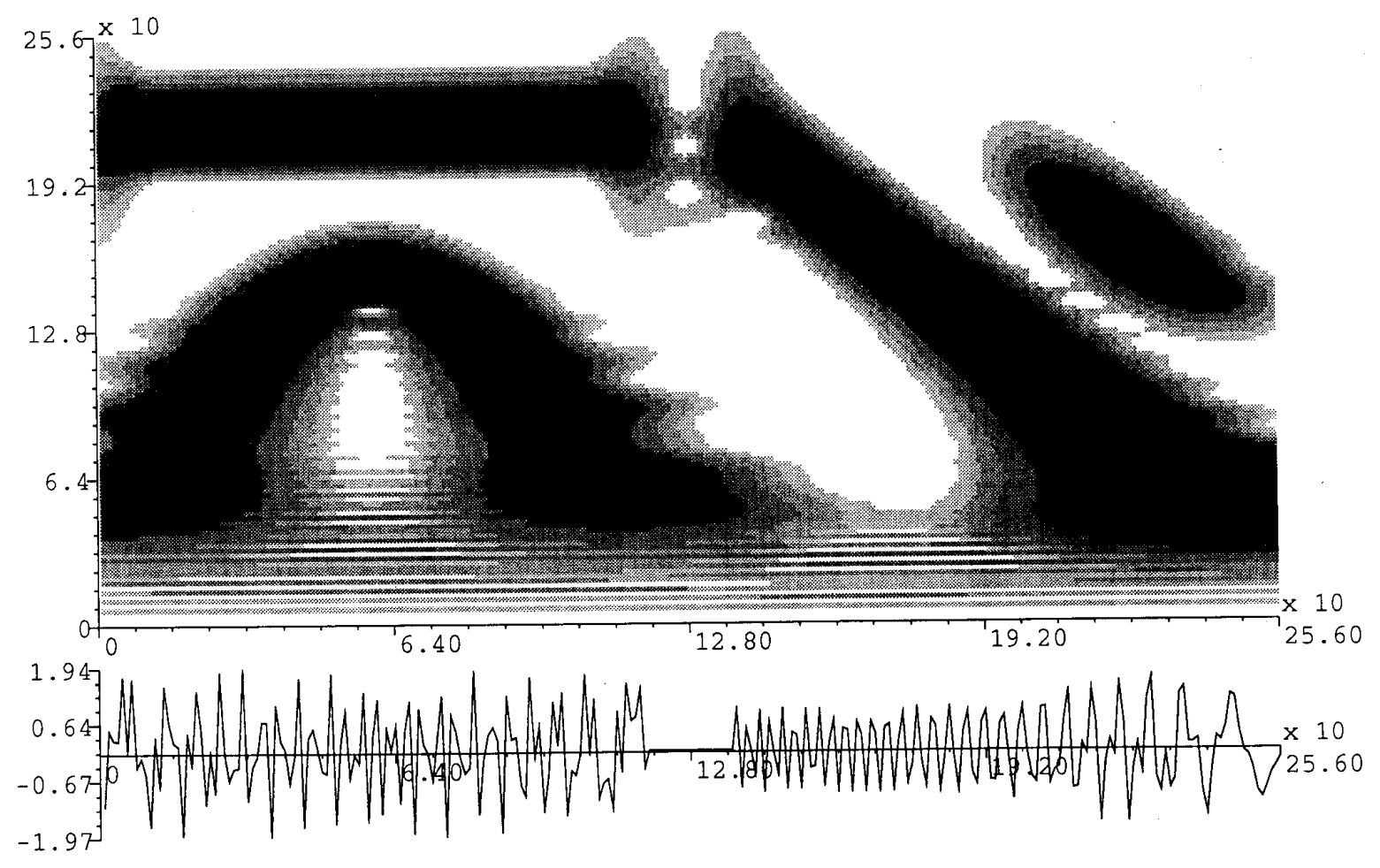

(a)

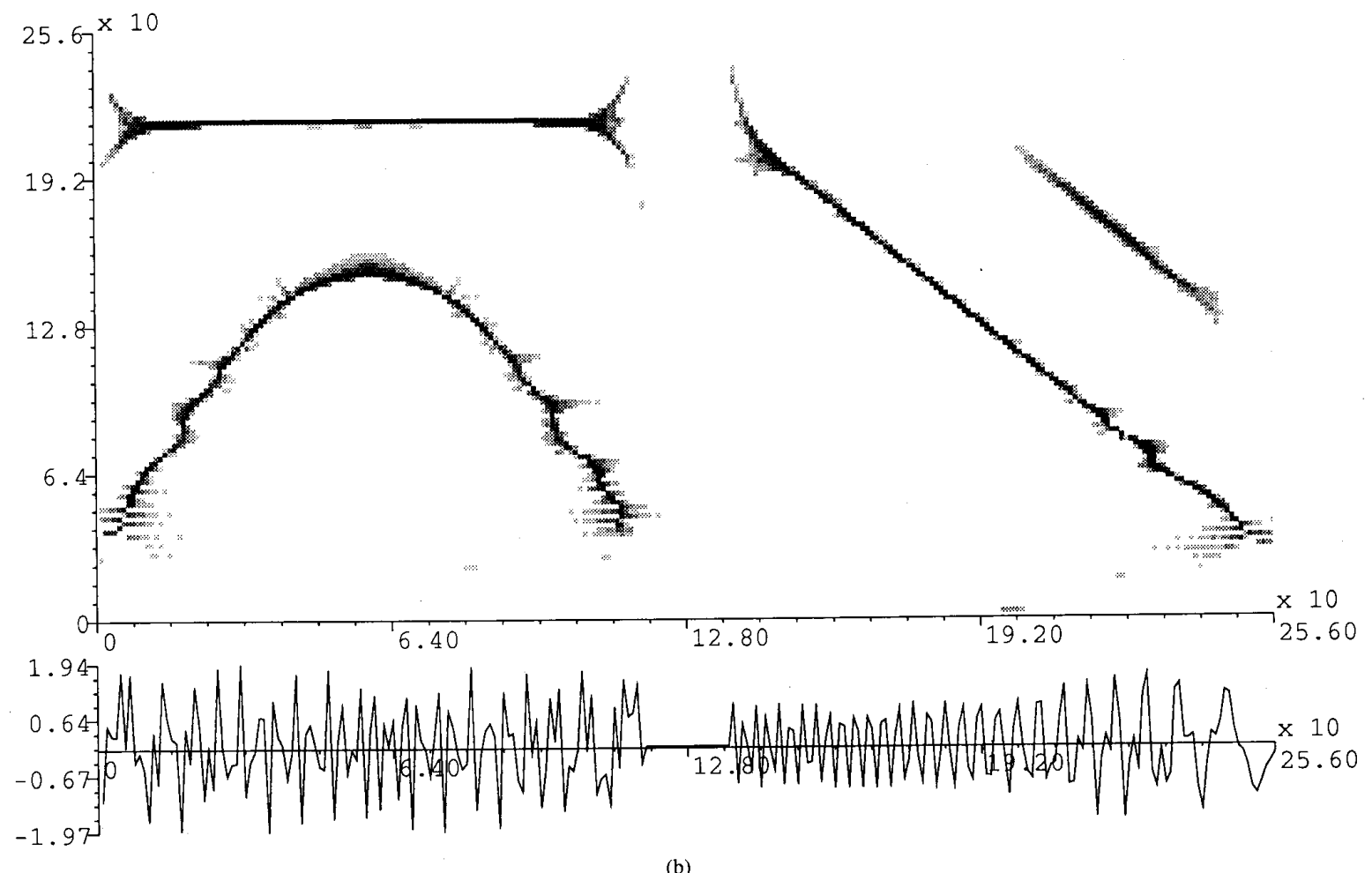

(b)

Fig. 13. (a) Scalogram of the signal, $h$ : Gaussian window with $F_{0} \cdot T h=3.0$. (b) Modified version of the scalogram shown in (a). 
TABLE II

Basic Properties of the Representations and of Their Modified Versions

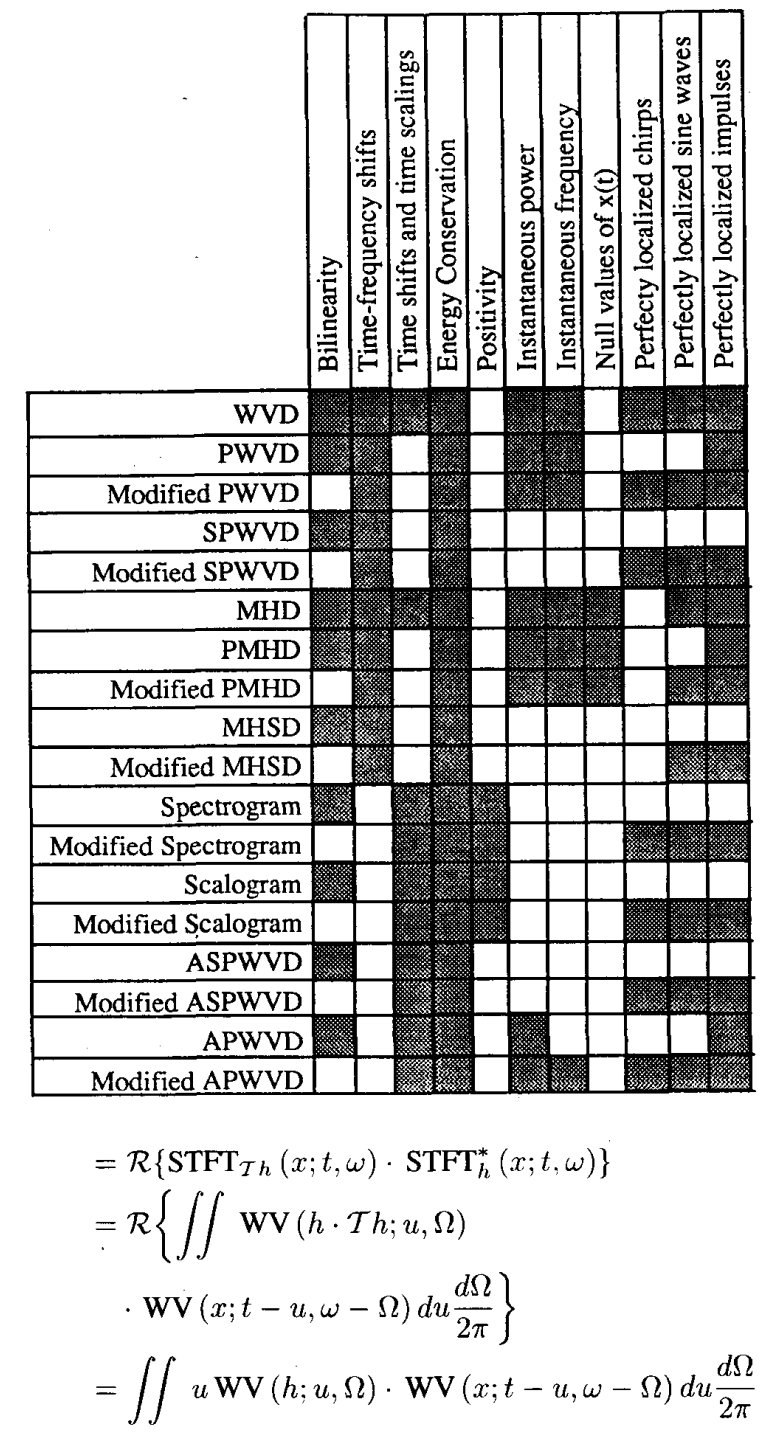

since

$$
\begin{aligned}
\mathcal{R}\{\mathrm{WV} & (h \cdot \mathcal{T} h ; u, \Omega) \\
= & \mathcal{R}\left\{u \mathrm{WV}(h ; u, \Omega)-\int \frac{\tau}{2} h(u+\tau / 2)\right. \\
& \left.\cdot h^{*}(u-\tau / 2) e^{-j \Omega \tau} d \tau\right\} \\
= & u \mathrm{WV}(h ; u, \Omega)
\end{aligned}
$$

For the frequency displacement:

$$
\begin{gathered}
\mathcal{R}\left\{\iint \Omega \cdot R i^{*}(h ; u, \Omega) \cdot R i(x ; t-u, \omega-\Omega) d u \frac{d \Omega}{2 \pi}\right\} \\
=\mathcal{R}\left\{\left(\int h^{*}(u) \cdot x(t-u) \cdot e^{-j \omega(t-u)} d u\right)\right. \\
\left.\cdot\left(\int \Omega H(\Omega) \cdot X^{*}(\omega-\Omega) e^{j \Omega t} \frac{d \Omega}{2 \pi}\right)\right\}
\end{gathered}
$$

$$
=\mathcal{R}\left\{-j \mathrm{STFT}_{h}\left(x ; t, \omega \cdot \mathrm{STFT}_{\mathcal{D} h}^{*}(x ; t, \omega\}\right.\right.
$$

$$
\begin{aligned}
= & -\mathcal{I} m\left\{\operatorname{STFT}_{\mathcal{D} h}(x ; t, \omega) \cdot \operatorname{STFT}_{h}^{*}(x ; t, \omega)\right\} \\
= & -\mathcal{I} m\left\{\iint \mathrm{WV}(h \cdot \mathcal{D} h ; u, \Omega)\right. \\
& \left.\cdot \mathrm{WV}(x ; t-u, \omega-\Omega) d u \frac{d \Omega}{2 \pi}\right\} \\
= & \iint \Omega \mathrm{WV}(h ; u, \Omega) \cdot \mathrm{WV}(x ; t-u, \omega-\Omega) d u \frac{d \Omega}{2 \pi}
\end{aligned}
$$

since

$$
\begin{aligned}
\operatorname{Im}\{\mathbf{W V}(h \cdot \mathcal{D} h ; u, \Omega)\} \\
=\operatorname{Im}\left\{-j \Omega \cdot \mathrm{WV}(h ; u, \Omega)+\int j \frac{\xi}{2} H(\Omega+\xi / 2)\right. \\
\left.\cdot H^{*}(\Omega-\xi / 2) e^{j \xi u} \frac{d \xi}{2 \pi}\right\} \\
=-\Omega \mathrm{WV}(h ; u, \Omega)
\end{aligned}
$$

Afterwards, the expressions (32a) and (32b) of the reassignment operators are derived from [35]:

$$
\begin{aligned}
&\left.\frac{\partial}{\partial t} \text { STFT }_{h}(x ; t, \omega)\right] \\
&= \frac{\partial}{\partial t}\left[M_{h}(x ; t, \omega)\right] \cdot e^{j \phi_{h}(x ; t, \omega)}+j \frac{\partial}{\partial t}\left[\phi_{h}(x ; t, \omega)\right] \\
& \cdot \operatorname{STFT}_{h}(x ; t, \omega) \\
&= \operatorname{STFT}_{\mathcal{D} h}(x ; t, \omega) \\
&\left.\frac{\partial}{\partial t} \operatorname{STFT}_{h}(x ; t, \omega)\right] \\
&=\frac{\partial}{\partial \omega}\left[M_{h}(x ; t, \omega)\right] \cdot e^{j \phi_{h}(x ; t, \omega)} \\
&+j \frac{\partial}{\partial \omega}\left[\phi_{h}(x ; t, \omega)\right] \cdot \operatorname{STFT}_{h}(x ; t, \omega) \\
&=-j t \operatorname{STFT}_{h}(x ; t, \omega)+j \operatorname{STFT}_{\mathcal{F} h}(x ; t, \omega) .
\end{aligned}
$$

\section{ACKNOWLEDGMENT}

The first author wishes to thank C. Doncarli, M. Guglielmi, and $\mathrm{J}$. J. Loiseau for providing suggestions and comments that have greatly improved the quality of this paper, and G. Garcia for his help and his American scientific language debugging.

\section{REFERENCES}

[1] D. Gabor, "Theory of communication," J. Inst. Electron. Eng., vol. 93. no. 11 , pp. 429-457, Nov. 1946.

[2] J. B. Allen and L. R. Rabiner. "A unified approach to short-time Fourier analysis and synthesis," in Proc. IEEE, vol. 65. 1977, pp. 1558-1566.

[3] L. Cohen. "Generalized phase space distribution functions," J. Math Phys., vol. 7, no. 5, pp. 781-786. 1966.

[4] B. Escudié and J. Gréa, "Sur une formulation générale de la représentation en temps et en fréquence dans I' analyse des signaux d'énergie finie." C. R. Acad. Sci. vol. 283, pp. 1049-1051. 1976 (in French).

[5] E. P. Wigner, "On the quantum correction for thermodynamic equilibrium," Phys. Rev., vol. 40, pp. 749-759, 1932.

[6] J. Ville, "Théorie et applications de la notion de signal analytique," Câbles et Transmissions, vol. 2A. pp. 66-74, 1948 (in French).

[7] H. Margenau and R. N. Hill. "Correlation between measurements in quantum theory," Prog. Theor. Phys., vol. 26. pp. 772-738, 1961.

[8] T. A. C. M. Claasen and W. F. G. Mecklenbräuker, "The Wigner distribution, a tool for time-frequency analysis, Part I: Continuous-time signals." vol. 35. no. 3, pp. 217-250: "Part II: Discrete-time signals," 
vol. 35 , no. 4/5, pp. 276-300; "Part III: Relations with other timefrequency signal transformations," Philips J. Res., vol. 35, no. 6, pp. $372-389,1980$

[9] P. Flandrin and W. Martin, "A general class of estimators for the Wigner-Ville spectrum of nonstationary processes," in Systems Analysis and Optimization of Systems, Lecture Notes in Control and Information Sciences. Berlin, Vienna, New York: Springer-Verlag, 1984, pp. 15-23.

[10] R. D. Hippenstiel and P. M. de Oliveira, "Time varying spectral estimation using the instantaneous power spectrum (IPS)," IEEE Trans. Acoust., Speech, Signal Processing, vol. 38, pp. 1752-1759, 1990.

[11] F. Auger and C. Doncarli, "Quelques commentaires sur des représentations temps-fréquence proposées récemment," Traitement $d u$ Signal, vol. 9, no. 1, pp. 3-25, 1992 (in French).

[12] M. Born and P. Jordan, "Zur Quantenmechanik," Z. Phys., vol. 34, pp. $858-888,1925$.

[13] H. I. Choi and W. J. Williams, "Improved time-frequency representation of multicomponent signals using exponential kernels," IEEE Trans. Acoust. Speech, Signal Processing, vol. 37, pp. 862-871, 1989.

[14] A. Papandreou and G. F. Boudreaux-Bartels, "Distributions for timefrequency analysis: A generalization of Choi-Williams and the Butterworth distributions," in Proc. IEEE ICASSP, vol. 5, pp.181-184, 1992.

[15] J. Jeong and W. J. Williams, "Kernel design for reduced interference distributions," IEEE Trans. Signal Processing, vol. 40, pp. 402-412, 1992.

[16] P. Goupillaud, A. Grossmann, and J. Morlet, "Cycle-octave and related transforms in seismic signal analysis," Geoexploration, vol. 23, pp. 85-102, 1984.

[17] I. Daubechies, "The wavelet transform, time-frequency localization, and signal analysis," IEEE Trans. Inform. Theory, vol. 36, pp. 961-1005, 1990.

[18] O. Rioul and P. Flandrin, "Time-scale energy distributions: A general class extending wavelet transforms," IEEE Trans. Signal Processing, vol. 40, pp. $1746-1757,1992$.

[19] J. Bertrand and P. Bertrand, "Time-frequency representations of broadband signals," in Proc. IEEE ICASSP, 1988, pp. 2196-2199.

[20] L. Cohen, "Time-frequency distributions-A review," in Proc. IEEE, vol. 77,1989 , pp. 941-981.

[21] F. Hlawatsch and G. F. Boudreaux-Bartels, "Linear and quadratic timefrequency signal representations," IEEE Signal Processing Mag., pp. 21-67, Apr. 1992

[22] W. F. G. Mecklenbräuker, Ed., The Wigner Distribution, Theory and Applications in Signal Processing. Amsterdam, The Netherlands: Elsevier, 1994.

[23] P. Flandrin, "Représentation temps-fréquence des signaux nonstationnaires," Thèse de doctorat d'état, INPG, 1987 (in French).

[24] F. Auger, "Représentations temps-frequence des signaux nonstationnaires: Synthèse et contributions," thèse de doctorat, Ecole Centrale de Nantes, 1991 (in French).

[25] F. Hlawatsch and P. Flandrin, "The interference structure of the Wigner distribution and related time-frequency signal representations," in The Wigner Distribution-Theory and Applications in Signal Processing, W. Mecklenbräucker, Ed. Amsterdam, The Netherlands: Elsevier, 1994.

[26] R. G. Baraniuk and D. L. Jones, "A radially Gaussian, signal dependent time-frequency representation," in Proc. IEEE ICASSP, 1991, pp. 3181-3184.

[27] S. Qian and J. M. Morris, "Wigner distribution decomposition and cross-terms deleted representation," Sig. Processing, vol. 27, no. 2, pp. $125-144,1992$

[28] S. Mallat and Z. Zhang, "Adaptive time-frequency decomposition with matching pursuits," in Proc. IEEE Sig. Processing Int. Symp. TimeFrequency and Time-Scale Anal., 1992, pp. 7-10.

[29] P. Duvaut and C. Jorand, "Analyse en composantes independantes d'un noyau multi-linéaire. Application a une nouvelle représentation tempsfréquence de signaux non-Gaussiens," $13^{\mathrm{eme}}$ Colloque GRETSI, pp. 61-64, 1991 (in French)

[30] M. Sun, C. C. Li, L. N. Sekhar, and R. J. Sclabassi, "Elimination of the cross-components of the discrete pseudo Wigner-Ville distribution via image processing," in Proc. IEEE ICASSP, 1989, pp. 2230-2233.

[31] H. Oung and J. M. Reid, "The Analysis of non-stationary Doppler spectrum using a modified Wigner-Ville distribution," Int. Conf. IEEE $E M B S$, vol. 12 , no. 1 , pp. $460-461,1990$

[32] F. Auger and C. Doncarli, "Un algorithme d'élimination des termes d'interférence de la transformée de Wigner-Ville," Colloque Gretsi 89 , pp. 99-102, 1989 (in French).
[33] K. Kodera, C. de Villedary, and R. Gendrin, "A new method for the numerical analysis of time-varying signals with small BT values," Phys. Earth Planet. Interiors, no. 12, pp. 142-150, 1976.

[34] K. Kodera, R. Gendrin, and C. de Villedary, "Analysis of time-varying signals with small BT values," IEEE Trans. Acoust., Speech, Signal Processing, vol. ASSP-34, pp. 64-76, 1986.

[35] W. Rihaczek, "Signal energy distribution in time and frequency," IEEE Trans. Inform. Theory, vol. IT-14, pp. 369-374, 1968.

[36] D. H. Friedman, "Instantaneous frequency vs. time: An interpretation of the phase structure of speech," in Proc. IEEE ICASSP, 1985, pp. 29.10.1-4.

[37] S. M. Sussman, "Least squares synthesis of radar ambiguity functions," Trans. IRE, vol. 8, pp. 246-254, 1962

[38] F. Peyrin and R. Prost, "A unified definition for the discrete-time, discrete-frequency and discrete-time/frequency Wigner distributions," IEEE Trans. Acoust., Speech, Signal Processing, vol. ASSP-34, pp. $858-867,1986$

[39] J. Jeong and W. Williams, "Alias-free generalized discrete-time timefrequency distributions," IEEE Trans. Signal Processing, vol. 40, 1992.

[40] A. J. E. M. Janssen, "On the locus and spread of pseudo-density functions in the time-frequency plane," Philips J. Res., vol. 37, no. 3, pp. $79-110,1982$.

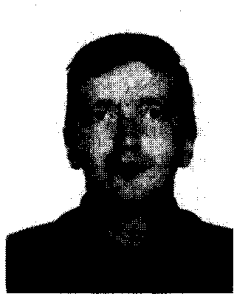

Francois Auger (M'93) was born in Saint Germain en Laye, France, in 1966. He received the Ingenieur degree and the D.E.A. in signal processing and automatic control from the Ecole Nationale Supérieure de Mécanique de Nantes in 1988, and the Doctorat from the Ecole Centrale de Nantes in 1991.

Since 1993, he has been Maître de Conférences at the IUT de Saint Nazaire. His current research interests include automatic control of power systems, spectral analysis and time-frequency representations.

Dr. Auger is a member of the GdR134 CNRS 'Traitement du Signal et des Images.

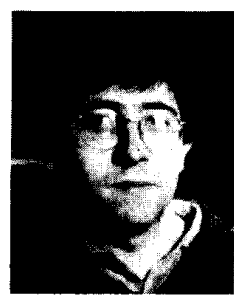

Patrick Flandrin (M'85) was born in Bron, France, in 1955. He received the Ingénieur degree from Institut de Chimie et Physique Industrielles de Lyon in 1978, the Docteur-Ingénieur degree and the Doctorat d'Etat ès Sciences Physiques from Institut National Polytechnique de Grenoble in 1982 and 1987, respectively.

He joined the Centre National de la Recherche Scientifique (CNRS) in 1982, where he is currently Directeur de Recherche. Until 1990, he was with ICPI Lyon, where he was Head of the Signal Processing Department from 1987 to 1990 . He is now with the Physics Department at Ecole Normale Supérieure de Lyon, in charge of the signal processing group. $\mathrm{He}$ is also leader of a working group within the CNRS cooperative structure "GdR Traitement du Signal et Images." His research interests include mainly nonstationary signal processing (with emphasis on time-frequency and time-scale methods), the study of self-similar stochastic processes and the analysis of chaotic signals and systems. He is the author of the book Temps-Fréquence (Paris: Hermès, 1993).

Dr. Flandrin has been a guest co-editor of the Special Issue "Wavelets and Signal Processing" of the IEEE Transactions on Signal Processinc and the Technical Program Chairman of the 1994 IEEE-SP International Symposium on Time-Frequency and Time-Scale Analysis. He is currently Associate Editor for the IEEE TRANSActions on Signal. Processing and Applied and Computational Harmonic Analysis, and a member of the Digital Signal Processing Committee of the IEEE Signal Processing Society. He was awarded the Philip Morris Scientific Prize in Mathematics in 1991, and is a member of the GdR134 CNRS 'Traitement du Signal et des Images. 